ARTICLE

\title{
Long noncoding RNA AGPG regulates PFKFB3- mediated tumor glycolytic reprogramming
}

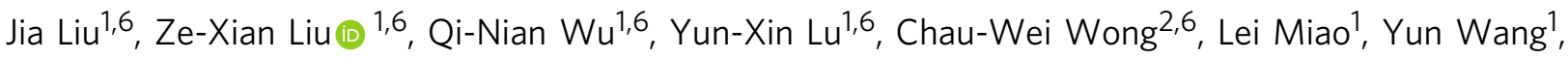 \\ Zixian Wang ${ }^{1}$, Ying Jin ${ }^{1}$, Ming-Ming He ${ }^{1}$, Chao Ren ${ }^{1}$, De-Shen Wang ${ }^{1}$, Dong-Liang Chen ${ }^{1}$, Heng-Ying Pu',

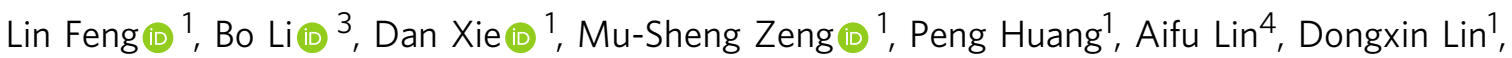 \\ Rui-Hua Xu (i) ${ }^{1,5 \bowtie} \&$ Huai-Qiang Ju (i) ${ }^{1,5}$ 凶
}

Tumor cells often reprogram their metabolism for rapid proliferation. The roles of long noncoding RNAs (IncRNAs) in metabolism remodeling and the underlying mechanisms remain elusive. Through screening, we found that the IncRNA Actin Gamma 1 Pseudogene $(A G P G)$ is required for increased glycolysis activity and cell proliferation in esophageal squamous cell carcinoma (ESCC). Mechanistically, AGPG binds to and stabilizes 6-phosphofructo-2-kinase/fructose-2,6-biphosphatase 3 (PFKFB3). By preventing APC/C-mediated ubiquitination, AGPG protects PFKFB3 from proteasomal degradation, leading to the accumulation of PFKFB3 in cancer cells, which subsequently activates glycolytic flux and promotes cell cycle progression. AGPG is also a transcriptional target of p53; loss or mutation of TP53 triggers the marked upregulation of $A G P G$. Notably, inhibiting AGPG dramatically impaired tumor growth in patient-derived xenograft (PDX) models. Clinically, AGPG is highly expressed in many cancers, and high AGPG expression levels are correlated with poor prognosis, suggesting that $A G P G$ is a potential biomarker and cancer therapeutic target.

\footnotetext{
${ }^{1}$ State Key Laboratory of Oncology in South China, Collaborative Innovation Center for Cancer Medicine, Sun Yat-sen University Cancer Center, Guangzhou 510060, China. ${ }^{2}$ The First Affiliated Hospital of Sun Yat-sen University, Guangzhou 510080, China. ${ }^{3}$ Department of Biochemistry and Molecular Biology, Zhongshan School of Medicine, Sun Yat-Sen University, Guangzhou 510080, China. ${ }^{4}$ College of Life Sciences, Zhejiang University, Hangzhou 310058, China. ${ }^{5}$ Precision Diagnosis and Treatment for Gastrointestinal Cancer, Chinese Academy of Medical Sciences, Guangzhou 510060, China. ${ }^{6}$ These authors contributed equally: Jia Liu, Ze-Xian Liu, Qi-Nian Wu, Yun-Xin Lu, Chau-Wei Wong. ${ }^{凶}$ email: xurh@sysucc.org.cn; juhq@sysucc.org.cn
} 
$\mathrm{R}$ apid proliferation and glucose metabolism remodeling are hallmarks of cancer. To provide sufficient energy and support rapid biosynthesis, cancer cells exhibit enhanced glycolysis, even under normoxic conditions; this phenomenon is referred to as the Warburg effect ${ }^{1}$. During glycolysis, glycolytic intermediates can be diverted to the biosynthesis of macromolecules, including nucleotides, amino acids, and fatty acids, which are necessary for cancer cell proliferation and tumor progression. Characterizing the cooperative mechanisms underlying glycolysis and cell proliferation could lead to a better understanding of human cancer development. Long noncoding RNAs (lncRNAs) are suggested to be involved in metabolic reprogramming, but the mechanisms remain elusive $^{2,3}$. Our recent studies investigated the roles of metabolic reprogramming in promoting glycolysis and redox hemostasis $^{4-7}$. The roles of lncRNAs in metabolism remodeling and the underlying mechanisms have thus attracted our interest.

Phosphofructo-2-kinase/fructose-2,6-biphosphatase 3 (PFKFB3) catalyzes the production of fructose-2,6-bisphosphate (F-2,6-BP), a potent allosteric stimulator of the key enzyme 6phosphofructokinase 1 . Thus, the activation of PFKFB3 has been linked to enhanced glycolysis ${ }^{8}$. Interestingly, PFKFB3 is mainly localized in the nucleus ${ }^{9}$, which is different from other members of the PFKFB family. Recent studies revealed the unexpected role of PFKFB3 in promoting cell proliferation by regulating the expression of important cell cycle proteins: cyclin-dependent kinase-1 (CDK1) is upregulated, and p27 is downregulated, partially owing to the nuclear delivery of $\mathrm{F}-2,6-\mathrm{BP}^{10}$. As reported, PFKFB3 inhibition is a promising modality for cancer treatment because it suppresses glycolysis, proliferation, and metastasis in cancer cells ${ }^{11-13}$.

In recent years, many lncRNAs have been identified to regulate cancer metabolism, but the underlying mechanisms remain elusive. Here, we identified that the IncRNA Actin Gamma 1 Pseudogene $(A G P G)$ has a pivotal role in glucose metabolism remodeling and cell proliferation by enhancing PFKFB3 stability. Intriguingly, this is the first lncRNA shown to directly bind to and regulate PFKFB3. The AGPG-PFKFB3 interaction protects PFKFB3 from ubiquitination and proteasomal degradation, thus promoting glycolysis and cell cycle progression at the G1/S phase transition. We also demonstrated that p53 binds to the AGPG promoter and represses its transcription, indicating that $A G P G$ is a target of p53. Moreover, high $A G P G$ expression levels are correlated with poor overall survival in esophageal squamous cell carcinoma (ESCC), suggesting that AGPG may be a biomarker and therapeutic target for ESCC treatment.

\section{Results}

Identification of AGPG as a metabolism-related lncRNA. To find oncogenic lncRNAs that significantly affect ESCC development, we first identified lncRNAs that were more highly expressed in ESCC tissues than in paired adjacent normal tissues from The Cancer Genome Atlas (TCGA) database. Then, we sorted these lncRNAs according to the log2-fold change. Next, we built an siRNA library targeting the top 50 lncRNAs (Supplementary Fig. 1a). For the siRNA screening, the siRNA library was designed with the SMARTselection algorithm to ensure high-efficiency silencing. These siRNAs also contained the proprietary ON-TARGETplus dual-strand chemical modification to ensure optimal strand loading and to disrupt microRNA-like seed activity, thereby reducing off-target effects. To pinpoint lncRNAs that might alter glucose metabolism, we transfected the siRNA library into two human ESCC cells and examined cell viability and lactate production. We found 14 lncRNAs that might be required for cell proliferation, 10 involved in lactate production and 8 potentially involved in both cell viability and glucose metabolism (Fig. 1a). Among these eight lncRNAs, AGPG knockdown significantly decreased cell viability and lactate production (Fig. 1b, Supplementary Fig. 1b). Bioinformatics analysis revealed that $A G P G$ is located on chromosome $1 \mathrm{q} 32.1$ and has 3 exons (1-56, 10,447-10,526, and 11,304-13,488) (Supplementary Fig. 1c). We focused on the isoform AC098934.2-201, and for simplicity, we refer to this isoform as AGPG. According to the coding potential calculator, the coding potential of $A G P G$ is very low.

Then, we verified $A G P G$ expression levels in a panel of human ESCC cells and normal esophageal epithelial cells (Het-1A and NE-1). We found that $A G P G$ levels were significantly higher in the tumor cells than in the normal cells, and the copy number of AGPG was also increased in ESCC cells (Fig. 1c, d, Supplementary Fig. 1d). The functional role of $A G P G$ in cell proliferation and lactate production was further verified in other ESCC cell lines (Supplementary Fig. 1e-g).

AGPG expression correlates with prognosis of ESCC. Consistent with our bioinformatics analysis results (Fig. 1e), we found that high AGPG levels were correlated with an unfavorable overall survival in ESCC patients in an independent cohort (Fig. 1f; Sun Yat-sen University Cancer Center (SYSUCC), $n=122$; clinicopathological information is provided in Supplementary Table 1). We categorized gene expression as low or high in comparison with the median value: if the expression level was higher than the median, it was classified as high, whereas if it was lower than the median, it was low. Multivariate analysis also indicated that $A G P G$ was an independent prognostic factor in ESCC patients (Supplementary Table 2).

As suggested by the TCGA database analysis, AGPG was highly expressed in multiple types of cancer, including gastric cancer (GC), colorectal cancer (CRC), liver cancer, breast cancer, and lung cancer (Supplementary Fig. 1h). However, in some cancers, such as glioblastoma multiforme (GBM), head and neck squamous cell carcinoma and thyroid carcinoma, there was no significant difference in expression between tumor and normal tissues. In addition, $A G P G$ levels were decreased in cancers such as kidney chromophobe, kidney renal clear cell carcinoma, and kidney renal papillary cell carcinoma. Similar to many other lncRNAs, AGPG has tissue-specific expression patterns in different cancers ${ }^{14}$. Next, we performed qRT-PCR and RNAScope in situ hybridization (ISH) assays to detect $A G P G$ expression in ESCC, GC, and CRC tissues ${ }^{15}$. Our results showed that AGPG was highly expressed in ESCC, GC, and CRC tissues (Fig. 1g-i). These results suggest a strong relationship between AGPG dysregulation and cancer development.

To identify the subcellular localization of $A G P G$, we detected $A G P G$ expression in cytoplasmic and nuclear fractions by qRTPCR analysis. The results showed that $A G P G$ was localized predominantly in the nucleus, with some localization in the cytoplasm, which was further verified by RNAScope ISH and RNA fluorescence in situ hybridization assays ${ }^{16}$ (Fig. 1j-l, Supplementary Fig. 1i).

AGPG promotes cell proliferation and glycolysis. Because $A G P G$ is potentially involved in cell proliferation and lactate production, we further investigated the functional role of $A G P G$ in cellular behaviors. AGPG knockdown in KYSE150 and KYSE30 cells strikingly inhibited cell proliferation and colony formation (Fig. 2a-d, Supplementary Fig. 2a, b), and AGPG knockdown blocked the G1/S cell cycle transition (Fig. 2e, f, Supplementary Fig. 2c). We also detected key cell cycle proteins and observed that $A G P G$ knockdown markedly increased p27 expression and 
a

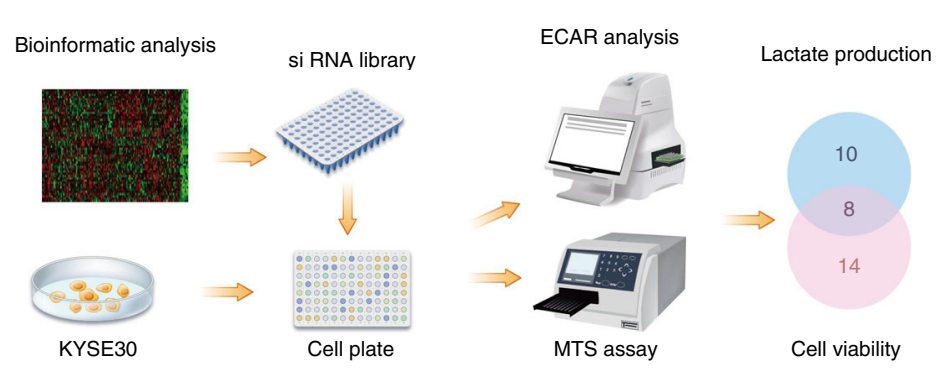

b

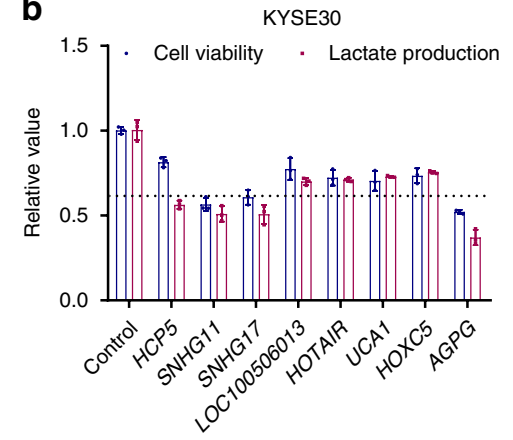

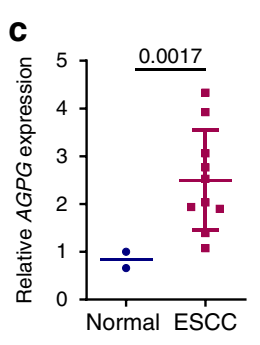

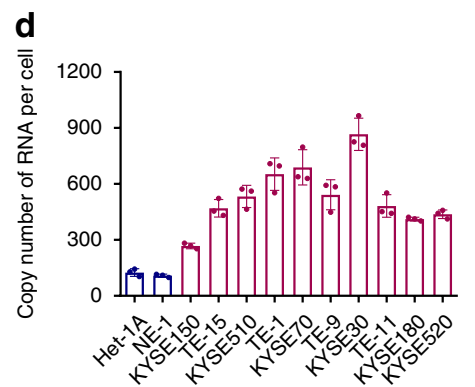

$\mathbf{g}$

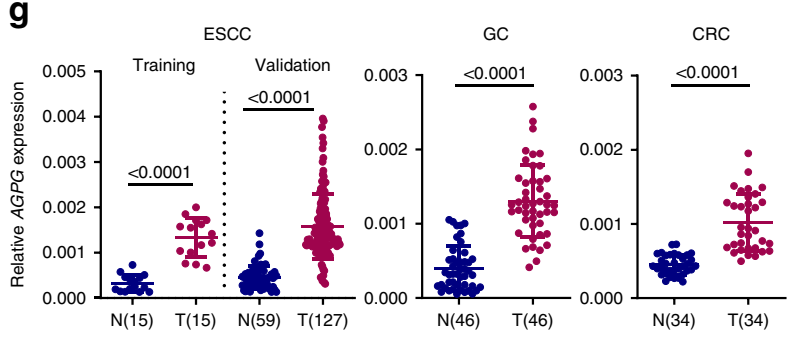

h

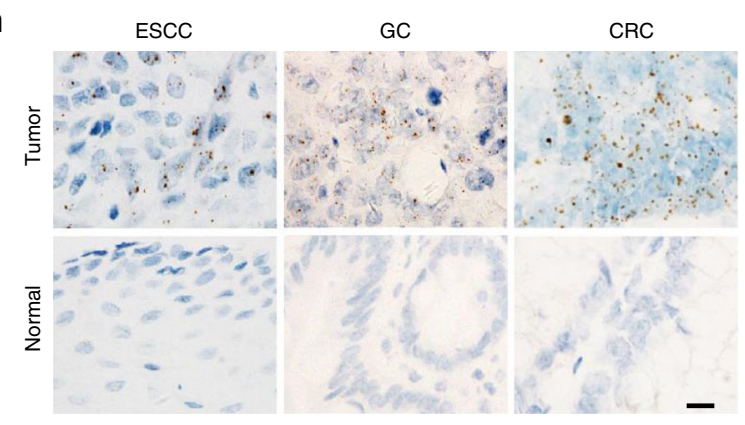

i

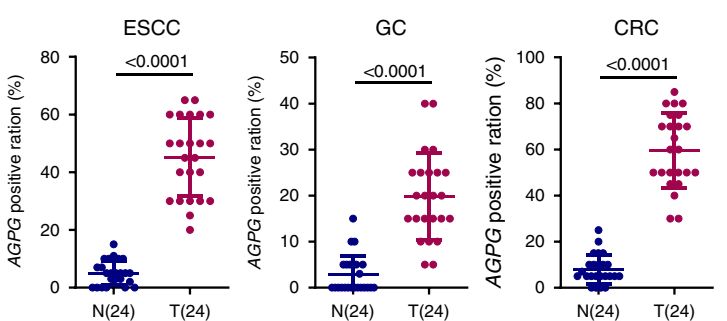

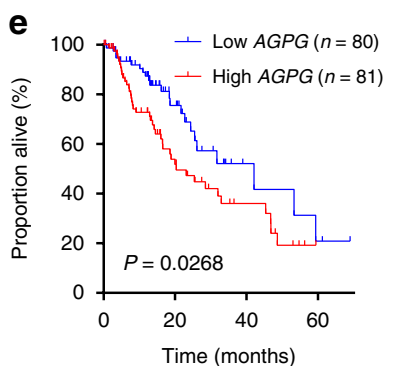

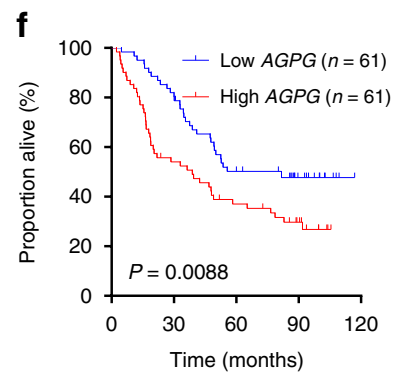

j

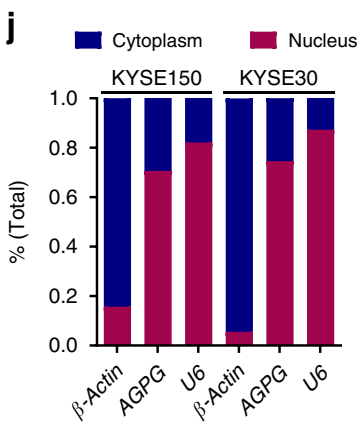

I

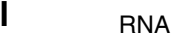

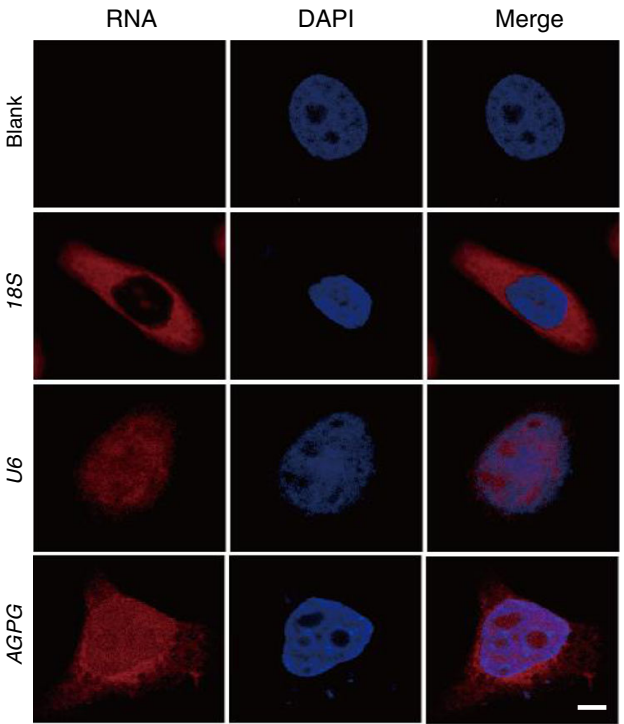

$\mathbf{k}$

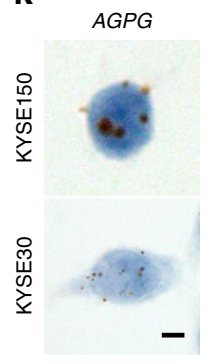

Merge decreased CDK1 expression (Fig. 2g) but did not change the expression of p21, p53, CDK3, or CDK6 (Supplementary Fig. 2d). These results suggest that $A G P G$ might regulate key cell cycle proteins, including $\mathrm{p} 27$, and CDK1-mediated G1/S progression to promote proliferation.

To verify the role of $A G P G$ in metabolic reprogramming, the extracellular acidification rate (ECAR) of ESCC cells was measured using a Seahorse XF24e Extracellular Flux Analyzer (Fig. 2h, i). We demonstrated that AGPG knockdown significantly impaired glycolysis, which was consistent with our previous screening results. To further determine the metabolic flux of glucose, we detected intracellular amounts of ${ }^{13} \mathrm{C}$-labeled metabolic intermediates in ESCC cells after incubation with ${ }^{13} \mathrm{C}_{6}$-glucose for $2 \mathrm{~h}$ (Fig. $2 \mathrm{j}$ ). Metabolome 
Fig. 1 Identification of AGPG as a metabolism-related IncRNA. a Experimental scheme for identifying IncRNAs potentially involved in both cell viability and glucose metabolism. $\mathbf{b}$ Eight IncRNAs regulated both cell proliferation and lactate production in KYSE30 cells, $n=3$ biologically independent samples. c qPCR detection of AGPG expression in multiple ESCC cells $(n=10$ cells) and in normal esophageal epithelial cell lines $(n=2$ cells). $\mathbf{d}$ Determination of AGPG copy number, $n=3$ biologically independent samples. e Overall survival analysis based on AGPG levels in ESCC (TCGA, $n=161$, log-rank test, twosided). $\mathbf{f}$ Overall survival analysis based on AGPG levels in ESCC detected by qPCR (SYSUCC, $n=122$, log-rank test, two-sided). $\mathbf{g}$ qPCR detection of $A G P G$ expression in ESCC (training set $n=15$, validation set $n=59,127$, respectively), GC $(n=46), C R C(n=34)$ and normal tissues. $\mathbf{h}$ RNAScope ISH detection of AGPG expression in ESCC, GC, CRC, and matched normal tissues. Scale bar: $20 \mu \mathrm{m}$. i RNAScope ISH detection and statistical analysis of AGPG expression in ESCC, GC, CRC, and matched normal tissues. Data are presented as mean \pm S.D., $n=24$ cases per tissue type, the $p$ value was determined by a two-tailed unpaired Student's $t$ test. $\mathbf{j}$ qPCR detection of AGPG expression in the cytoplasmic and nuclear fractions. $\mathbf{k}$ RNAScope ISH detection of $A G P G$ subcellular localization. Scale bar: $5 \mu \mathrm{m}$. I Subcellular localization of AGPG detected by FISH. Scale bar: $5 \mu \mathrm{m}$. Data in b-d, $\mathbf{g}$, $\mathbf{i}$ are representative of three independent experiments and presented as mean \pm S.D., the $P$ value was determined by a two-tailed unpaired Student's $t$ test.

analysis based on liquid chromatography and mass spectrometry (MS) showed that intracellular metabolites of glycolysis (3-phosphoglycerate, pyruvate, and lactate) were markedly decreased after $A G P G$ knockdown, further confirming that $A G P G$ is essential for the conversion of glucose to lactate ${ }^{17}$ (Fig. 2k-m).

To further confirm the functional role of $A G P G$, we generated AGPG CRISPR KO cells using the CRISPR/Cas9 genome-editing system (Supplementary Fig. 2e). Consistently, AGPG CRISPR KO significantly inhibited ESCC cell proliferation and cell cycle progression (Supplementary Fig. 2f, g). In addition, AGPG CRISPR KO led to a significant reduction in aerobic glycolysis (Supplementary Fig. 2h). Taken together, our data indicated that $A G P G$ is functionally important in regulating cancer metabolic reprogramming and tumor growth.

AGPG is directly associated with PFKFB3. To dissect the molecular mechanisms underlying $A G P G$-mediated metabolic remodeling, we tried to identify $A G P G$-associated proteins through RNA pull-down assays followed by mass spectrometry. The mass spectrometry data are provided in Supplementary Data 1,2 . We compared the $A G P G$-binding proteins with antisense $A G P G$-binding proteins. Proteins that bound to antisense $A G P G$ were excluded from the candidate list, and the remaining proteins were sorted by MS score, as described in previous lncRNA studies ${ }^{15}$. We found that sense $A G P G$, but not the antisense control, interacted specifically with PFKFB3 (Fig. 3a), which was also localized mainly in the nucleus, as previously reported. This observation was further confirmed by the finding that $A G P G$ bound directly to purified His-tagged recombinant PFKFB3 (Fig. 3b). The interaction between AGPG and PFKFB3 was also confirmed by RNA immunoprecipitation (RIP) assays (Fig. 3c, Supplementary Fig. 3a, b). To further characterize the interaction between AGPG and PFKFB3 in vivo, we performed MS2-tagged RNA affinity purification (MTRAP) and western blotting. Compared with expression of the negative control, coexpression of the MS2-AGPG and MCP-3FLAG plasmids led to significant enrichment of PFKFB3, demonstrating that PFKFB3 specifically binds to AGPG (Fig. 3d).

Immunofluorescence colocalization analysis showed that AGPG and PFKFB3 colocalized mainly in the nucleus, with some colocalization in the cytoplasm, which suggests that the $A G P G-P F K F B 3$ complex may play roles in both the nucleus and cytoplasm (Fig. 3e). Moreover, we examined AGPG expression by qPCR and PFKFB3 expression by western blotting in a panel of ESCC cells and 12 pairs of ESCC tissues and matched normal esophageal tissues (SYSUCC). As expected, AGPG expression was positively correlated with PFKFB3 expression in ESCC (Fig. 3f, Supplementary Fig. 3c), which further implied the functional relationship between $A G P G$ and PFKFB3. In addition, PFKFB3 was more highly expressed in ESCC tissues than in normal tissues, as previously reported.
Absolute quantification of $A G P G$ and PFKFB3 levels showed that there were $\sim 400-700$ AGPG molecules per cell versus 4400-7400 PFKFB3 molecules per cell (Supplementary Fig. 3d), indicating that there are sufficient $A G P G$ copies in ESCC cells ${ }^{18}$. Collectively, these results suggest that AGPG and PFKFB3 are closely related and that their interaction plays an important role in human cancer development.

The T5 fragment of $A G P G$ mediates the interaction with PFKFB3. Computational secondary structure analysis revealed that $A G P G$ contains five main branches (Supplementary Fig. 3e). To map the regions that mediate the interaction of $A G P G$ with PFKFB3, we performed RNA pull-down assays using in vitrosynthesized full-length (FL) AGPG and T1 (1-800), T2 (801-1140), T3 (1141-1700), T4 (1701-2030), and T5 (2031-2321) fragments and then analyzed the products by western blotting. We demonstrated that the T5 fragment could bind to PFKFB3, whereas the other fragments or the beads-only control could not. These results were further verified with purified recombinant PFKFB3 (Fig. 3g). We also performed crosslinking IP and qPCR (CLIP-qPCR), an improved method for the isolation of lncRNA segments bound by PFKFB3; consistently, the T5 fragment was identified as the main region responsible for binding PFKFB3 (Fig. 3h, Supplementary Fig. 3f).

After deleting the T5 fragment, $A G P G$ could no longer interact with PFKFB3 (Fig. 3i). Overexpression of $A G P G$ FL, but not a mutant lacking the T5 fragment ( $A G P G \Delta \mathrm{T} 5$ ), was sufficient to prevent the phenotypes observed after $A G P G$ knockout (KO), including glycolytic reprogramming and cell proliferation (Fig. 3j, k, Supplementary Fig. 3g, h). These results suggest that the T5 fragment is required for $A G P G$ to interact with and regulate PFKFB3, and the downstream cellular processes are probably mediated through the interaction of $A G P G$ with PFKFB3.

To further identify the specific motif that are responsible for PFKFB3 binding, we performed crosslinking-IP and highthroughput sequencing (HITS-CLIP). Hypergeometric Optimization of Motif EnRichment (HomeR) was used for motif analysis based on the binding peaks obtained by Piranha and CIMS analyses ${ }^{19,20}$. The RNA motifs recognized by PFKFB3 are listed in Supplementary Table 3. Among these motifs, CCAGCCA or similar motifs were highly ranked and could be identified by multiple methods (Fig. 31). Compared with the input, PFKFB3 CLIP enriched more reads mapping to the $A G P G$ sequence around the identified motif. To further verify whether this motif coordinates PFKFB3 binding, we performed RNA pull-down assays using wild-type $A G P G$ (WT) and CCAGCCA motif-deleted $A G P G$ (MT). We demonstrated that the binding of a mutant lacking the CCAGCCA motif (AGPG MT) and PFKFB3 was significantly reduced (Supplementary Fig. 3i). In addition, overexpression of this AGPG MT could not rescue the decreased glycolysis caused by $A G P G$ KO (Supplementary Fig. 3j). These data suggest that the CCAGCCA motif of AGPG 
a

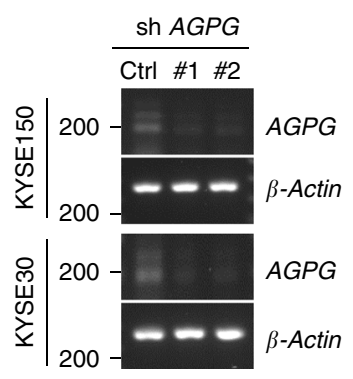

b

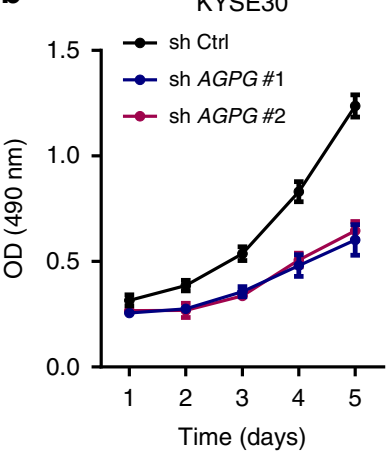

c

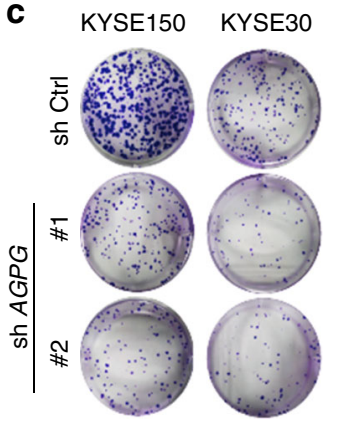

d $\quad \cdot$ sh Ctrl

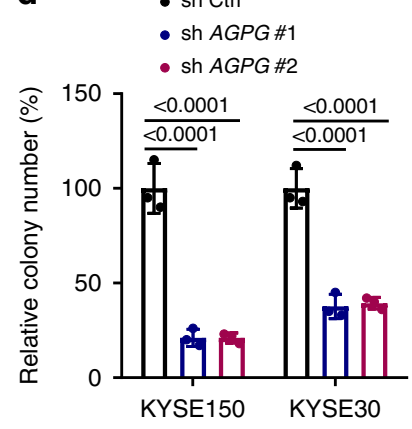

e

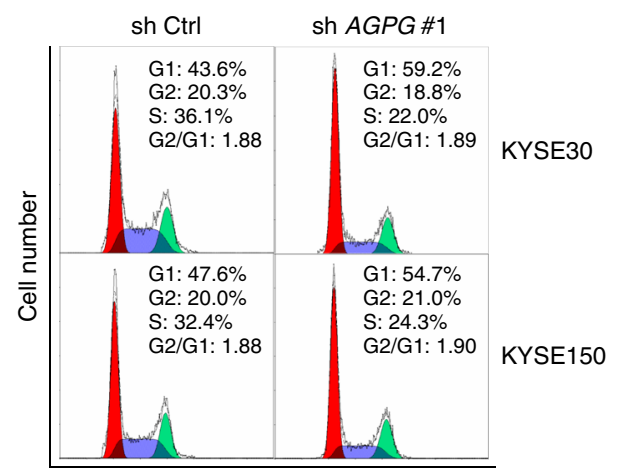

DNA content-PE

h

$$
\text { KYSE150 }
$$

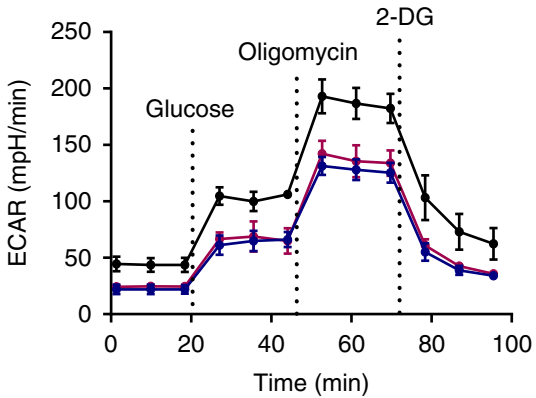

j

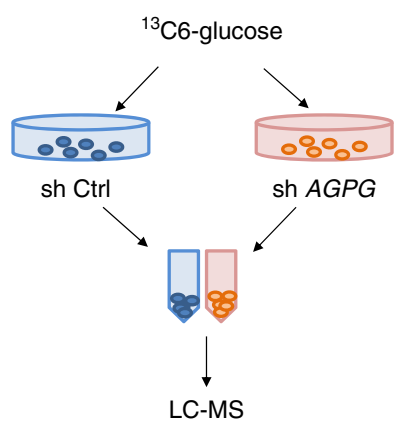

k

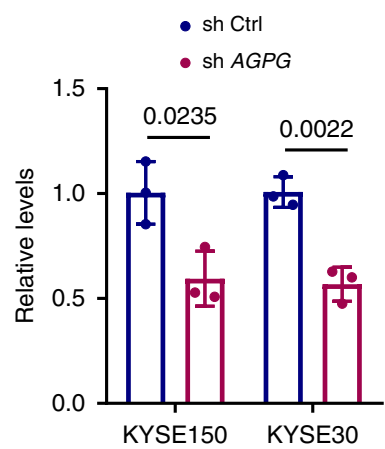

f

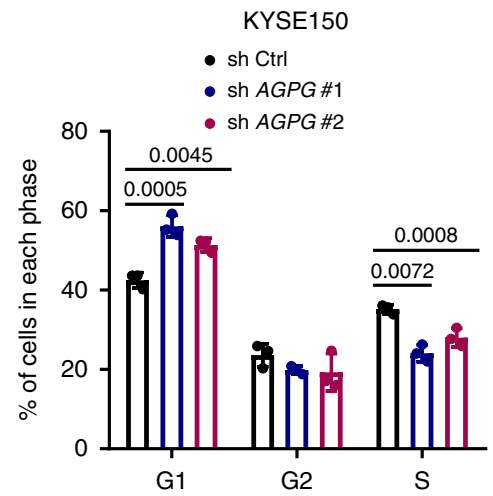

g
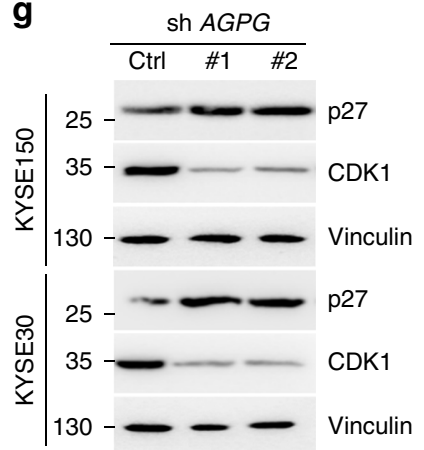

- sh Ctrl

i $\quad$ - sh $A G P G \# 1$

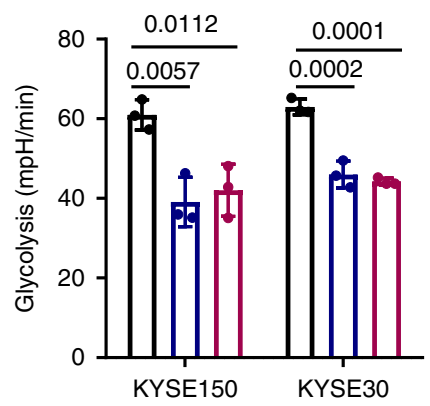

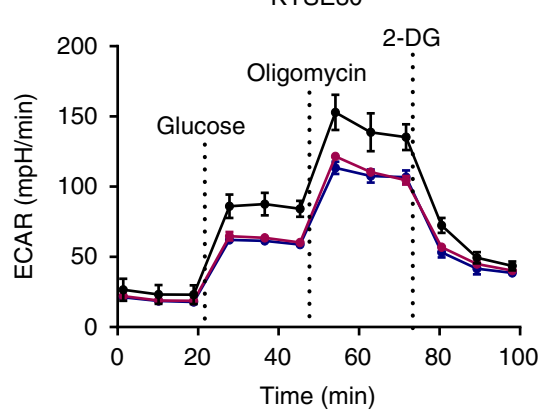

I $\quad{ }^{13} \mathrm{C}$ labelled pyruvate

m
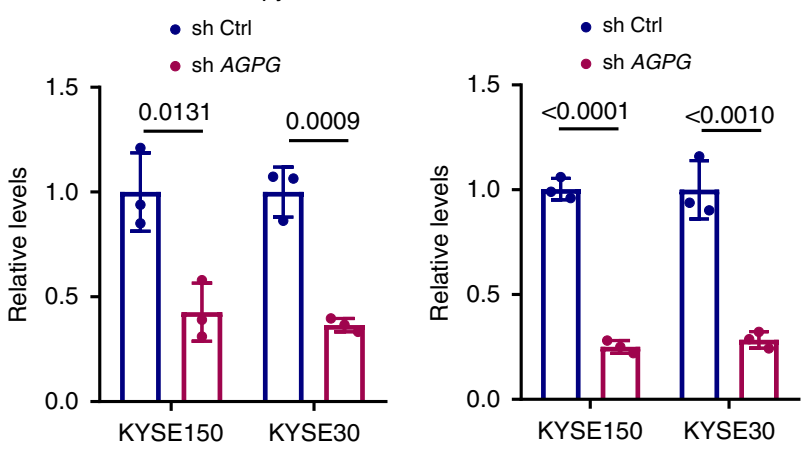

is important for its ability to bind PFKFB3 and promote tumor glycolytic reprogramming.

AGPG blocks APC/C-mediated PFKFB3 ubiquitination. Because PFKFB3 contains a kinase domain and a phosphatase domain, three human FLAG-tagged PFKFB3 vectors harboring

FL (1-520), N-terminal (N, 1-245) and C-terminal (C, 246-520) constructs were constructed to identify the PFKFB3 residues that associated with $A G P G$. Interestingly, we demonstrated that $A G P G$ interacted mainly with the C-terminal fragment, with minimal binding with the N-terminal fragment (Fig. 4a, Supplementary Fig. 4a). Then, we performed RIP assays using an anti-FLAG 
Fig. 2 AGPG is required for cell proliferation and metabolism remodeling. a qPCR and electrophoresis detection of AGPG expression in KYSE30 and KYSE150 cells. Ctrl, control. b Cell proliferation was assessed by MTS assays (OD $490 \mathrm{~nm}$ ). c, d Colony formation assays and statistical analysis of ESCC cells transduced with shAGPG \#1 or \#2 or shCtrl. e The cell cycle was analyzed by flow cytometry analysis. f Statistical analysis of KYSE150 cells (\%) in each cell cycle phase. $\mathbf{g}$ CDK1 and p27 expression levels were detected by western blotting in cells transfected with shAGPG \#1 or \#2 or shCtrl. $\mathbf{h}$ The ECAR was measured in cells transfected with shAGPG \#1 or \#2 or shCtrl using an XF Extracellular Flux Analyzer. $\mathbf{i}$ Statistical analysis of the effects of AGPG knockdown on glycolytic activity. j Flowchart of the experiments for identifying the role of AGPG in glucose metabolism. $\mathbf{k}$-m ${ }^{13} \mathrm{C}$-Labeled metabolic intermediates of glycolysis were decreased after AGPG knockdown. Data in $\mathbf{b}, \mathbf{d}, \mathbf{f}, \mathbf{i}, \mathbf{k}-\mathbf{m}$ are representative of three independent experiments and presented as mean \pm S.D., $n=3$ biologically independent samples, the $P$ value in $\mathbf{b}, \mathbf{d}, \mathbf{f}, \mathbf{i}$ was determined by one-way analysis of variance (ANOVA) with Dunnett's multiple comparisons test, no adjustments were made for multiple comparisons. The $P$ value in $\mathbf{k}$ - $\mathbf{m}$ was determined by a two-tailed unpaired Student's $t$ test.

antibody or IgG control. As expected, $A G P G$ precipitated with the C-terminal fragment of PFKFB3 (Fig. 4b).

Then, we evaluated the functional effect of the $A G P G$ PFKFB3 interaction on PFKFB3. Interestingly, AGPG knockdown mediated by shRNA significantly reduced the expression of PFKFB3 (Fig. 4c), which was also confirmed in $A G P G$ CRISPR KO cells using CRISPR/Cas9 (Fig. 4d). In addition, overexpression of $A G P G$ FL but not $A G P G \triangle \mathrm{T} 5$ rescued the decreased PFKFB3 level induced by $A G P G$ deletion (Fig. $4 \mathrm{~d}$ ). These data suggest that $A G P G$ may regulate the PFKFB3 protein levels through the T5 fragment.

Previous studies have revealed that PFKFB3 is subjected to constant proteosomal degradation through polyubiquitination ${ }^{21}$. Therefore, we hypothesized that the observed effects might be attributable to proteasomal degradation, as decreased PFKFB3 levels were recovered by the proteasomal inhibitor MG-132 (Fig. 4e). In addition, AGPG knockdown had marked effects on PFKFB3 stabilization in ESCC cells, shortening the half-life of PFKFB3 (Fig. 4f, Supplementary Fig. 4b, c). Moreover, we carried out IP assays in cells expressing FLAG-tagged PFKFB3 with an anti-FLAG antibody and detected ubiquitin levels by western blotting. As shown, AGPG knockdown significantly increased the levels of ubiquitinated PFKFB3 (Fig. 4g, Supplementary Fig. 4d). These data suggest that $A G P G$ is required for PFKFB3 stabilization. In addition, $A G P G$ CRISPR KO did not affect PFKFB3 mRNA levels or subcellular location (Supplementary Fig. 4e, f). Regarding PFKFB3 enzymatic activity, AGPG CRISPR KO had a mild effect on PFKFB3 S461 phosphorylation (Supplementary Fig. 4g), which is widely reported to be a key regulator of PFKFB3 enzyme activity $^{22}$. Therefore, we speculate that AGPG has little effect on PFKFB3 enzyme activity. Taken together, these data indicate that $A G P G$-PFKFB3 binding appears to be important for PFKFB3 protein turnover.

PFKFB3 contains a KEN box that targets proteins for ubiquitylation by the anaphase-promoting complex $\mathrm{APC} / \mathrm{C}^{21}$. PFKFB3 was shown to be subject to degradation involving APC/ C-Cdh1, a cell cycle-regulated E3 ubiquitin ligase. APC/C is composed of multiple subunits; as reported previously, active APC/ $\mathrm{C}$ could be immunoprecipitated from cells using a monoclonal Cdc27 antibody ${ }^{21,23}$. Therefore, to further elucidate the mechanism by which AGPG blocks PFKFB3 ubiquitination, we performed coIP assays using PFKFB3 and Cdc27 antibodies to determine whether $A G P G$ affects the interaction between PFKFB3 and active APC/C. $A G P G$ CRISPR KO significantly increased the PFKFB3/Cdc27 interaction (Fig. 4h), suggesting that $A G P G$ could block the binding of Cdc27 to PFKFB3. Therefore, we speculate that AGPG specifically binds to PFKFB3 and blocks its interaction with APC/ $\mathrm{C}$, which inhibits APC/C-mediated PFKFB3 ubiquitination and the subsequent degradation.

AGPG stabilizes PFKFB3 by preventing K302 ubiquitination. Because $A G P G$ binds mainly to the C-terminal fragment of PFKFB3, we analyzed four putative ubiquitinated lysine (K) residues, namely, K292, K302, K352, and K472, in the Cterminus of PFKFB3 to identify the predominant lysine residue (s) subject to ubiquitination that is (are) affected by AGPG (Supplementary Fig. 4h). We mutated these four lysine residues to alanine (A) and performed IP and RNA pull-down assays. Among the four mutants, PFKFB3 K302A showed no increase in ubiquitination in response to $A G P G$ knockdown (Fig. $4 \mathrm{i}$, Supplementary Fig. 4i), suggesting that $A G P G$ might stabilize PFKFB3 by preventing K302 ubiquitination. Moreover, PFKFB3 $\mathrm{K} 302 \mathrm{~A}$, but not other mutants, significantly abrogated APC/C (Cdc27)-induced PFKFB3 ubiquitination in ESCC cells (Supplementary Fig. 4j, k), further indicating that $\mathrm{K} 302$ is an important site for APC/C-mediated ubiquitination in PFKFB3. Thus, AGPG stabilizes PFKFB3 by preventing APC/C-mediated PFKFB3 K302 ubiquitination.

Furthermore, to ascertain the function of K302 in regulating PFKFB3, FLAG-tagged PFKFB3 wild-type (WT) or K302A was exogenously expressed in ESCC cells; intriguingly, the PFKFB3 K302A mutant showed an extended half-life (Fig. 4j, Supplementary Fig. 4l), indicating that $\mathrm{K} 302$ is an important ubiquitination site responsible for PFKFB3 stability. Then, we investigated the role of PFKFB3 in AGPG-mediated cell proliferation and glycolytic reprogramming. Consistently, PFKFB3 K302A overexpression could significantly reverse the inhibition of glycolysis, cell proliferation and cell cycle progression by AGPG CRISPR KO, whereas PFKFB3 WT could only partially rescue these effects (Fig. $4 \mathrm{k}-\mathrm{m}$, Supplementary Fig. $4 \mathrm{~m}, \mathrm{n}$ ). Taken together, these data show that by inhibiting PFKFB3 K302 ubiquitination, AGPG enhanced PFKFB3 stability and therefore led to the increased accumulation of PFKFB3 in cancer cells, thereby increasing F-2,6$\mathrm{BP}$ synthesis and subsequently promoting cell cycle progression by regulating p27 and CDK1. Simultaneously, the increased F-2,6-BP levels activated glycolytic flux by stimulating PFK-1 ${ }^{8,9}$. Furthermore, we tested the effect of $A G P G$ on PFKFB3 KO cell lines. After PFKFB3 KO in ESCC cells, AGPG CRISPR KO had mild effects on aerobic glycolysis and cell proliferation (Supplementary Fig. 5a, b). Collectively, these data suggest that the regulatory roles of AGPG in cell proliferation and glycolytic reprogramming are mainly dependent on PFKFB3.

AGPG is a transcriptional target of p53. Because $A G P G$ is highly expressed in tumors, we tried to determine the mechanism of $A G P G$ regulation. Pathway analysis indicated that $A G P G$ expression was negatively correlated with p53. (Fig. 5a). An analysis of cells with different TP53 genotypes showed that HCT116 cells with TP53 KO displayed higher AGPG levels than control cells (Fig. 5b), indicating an inhibitory effect of p53 on AGPG expression. Furthermore, cells with WT TP53 (KYSE150) expressed much lower AGPG levels than those harboring mutant (MT) TP53 (TE-1 and KYSE30) ${ }^{24}$ (Fig. 5c). WT TP53 overexpression in KYSE150 and HCT-116 cells decreased AGPG levels, whereas TP53 knockdown increased AGPG expression, further confirming the role of p53 in regulating $A G P G$ expression 
a

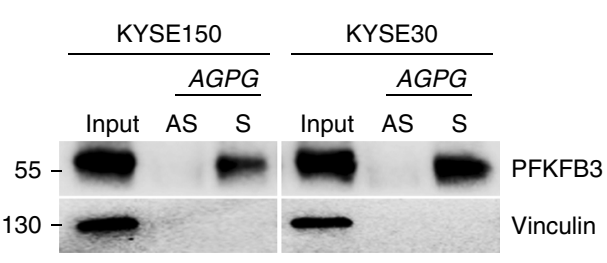

b

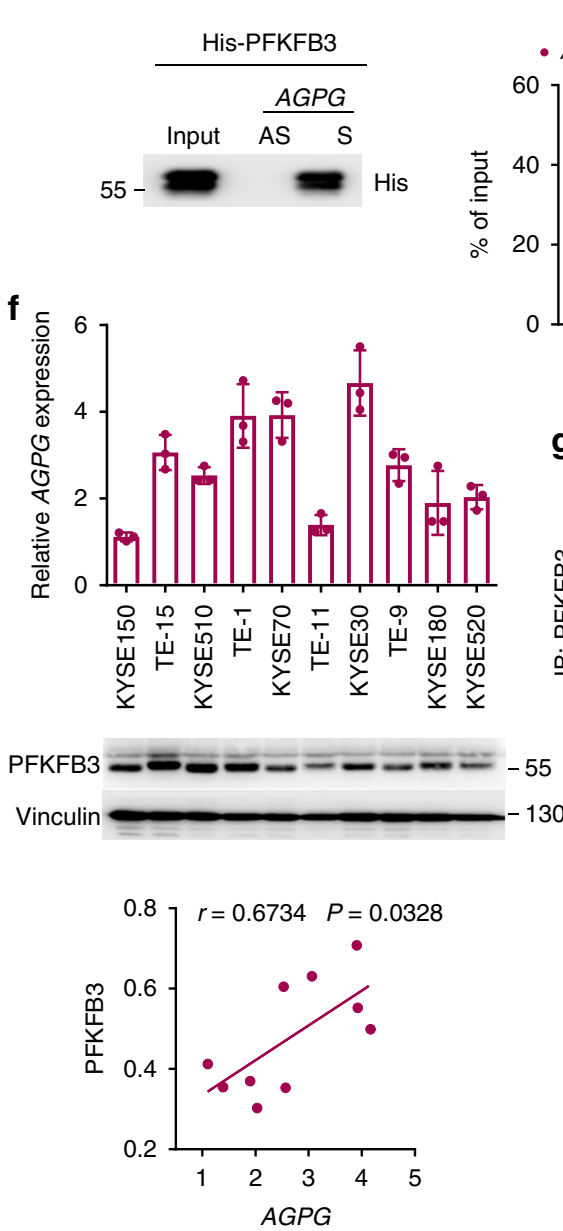

d

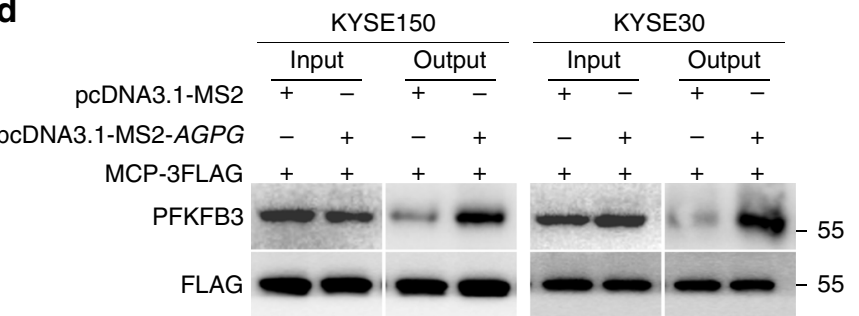

e

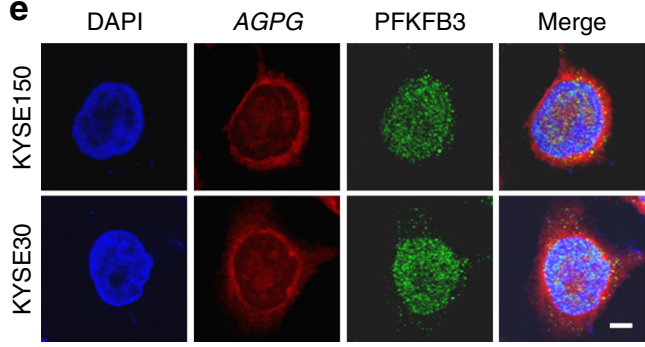

KYSE150

AGPG $\quad$ - $\beta$-Actin

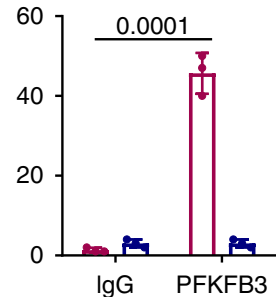

\section{g}

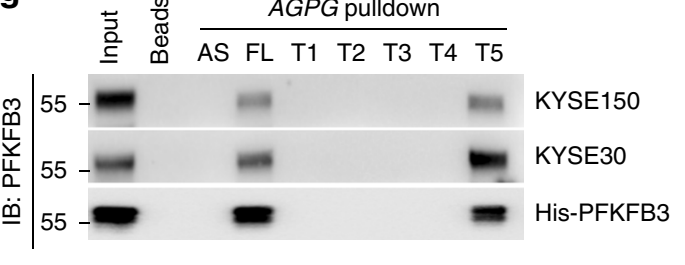

h

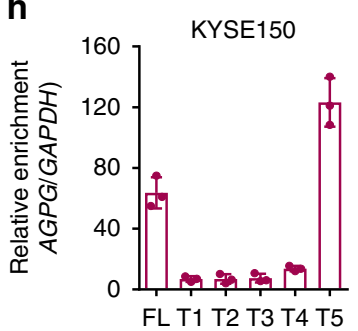

i

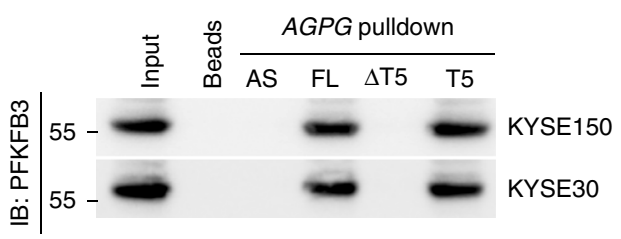

I

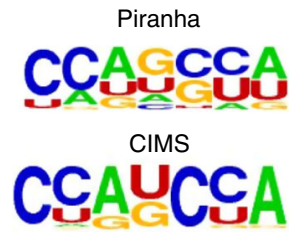

j

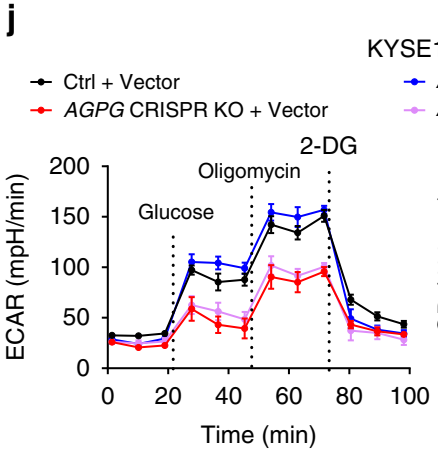

KYSE150

- AGPG CRISPR KO + AGPG FL - AGPG CRISPR KO + AGPG $\triangle \mathrm{T} 5$

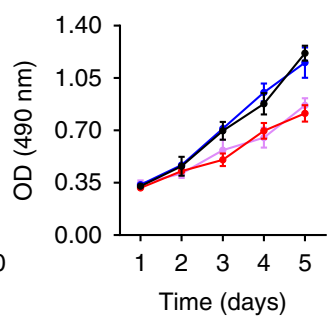

k

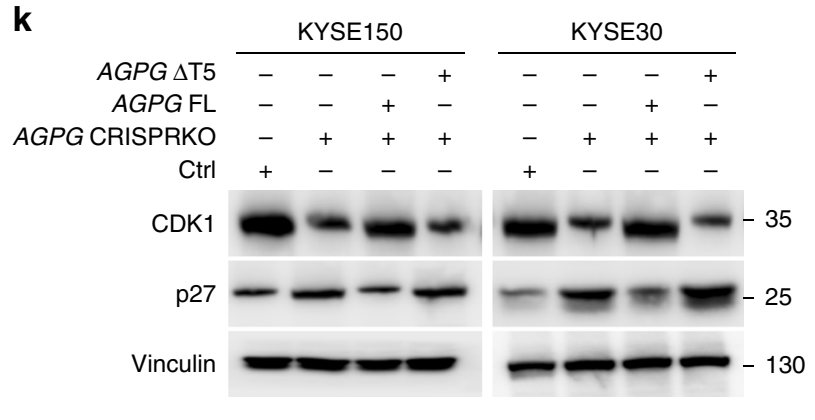

(Fig. 5d). Furthermore, AGPG expression level was negatively correlated with TP53 expression in a cohort of ESCC patients with WT TP53 by qPCR analysis (Fig. 5e, SYSUCC, $n=72$ ).

Next, we determined the region required for $\mathrm{p} 53$-mediated $A G P G$ regulation. As expected, the $A G P G$ promoter contains a p53-binding sequence (Fig. 5f), which was identified as a p53-binding region (p53-BR) according to chromatin immunoprecipitation (ChIP) assays ${ }^{25}$ (Fig. 5g, Supplementary Fig. 5c). Consistently, the transcriptional activity of luciferase reporters containing an intact $\mathrm{p} 53-\mathrm{BR}$ (p53-BR wt) was markedly weaker than that of those with the p53-BR deleted (p53-BR mt). Moreover, co-transfection of WT TP53 selectively decreased the 
Fig. 3 AGPG directly associates with PFKFB3. a, b PFKFB3 in cell lysates a or purified His-tagged recombinant PFKFB3 b was pulled down by biotin-labeled AGPG but not by AGPG antisense RNA. S, sense. AS, antisense. c RIP assays indicated that AGPG precipitated with PFKFB3 in whole-cell lysates. The RNA levels of $A G P G$ and $\beta$-actin were measured by qPCR analysis. $\mathbf{d}$ AGPG-binding proteins were detected by MTRAP and western blotting analysis. PFKFB3 bound to AGPG was captured by anti-FLAG antibody affinity agarose beads; IP complexes were separated and identified by specific antibodies. e Immunofluorescence analysis showed that AGPG and PFKFB3 colocalized not only in the nucleus but also in the cytoplasm. Scale bar: $5 \mu$ m. $\mathbf{f}$ qPCR detection of AGPG expression and western blotting detection of PFKFB3 expression in human ESCC cells. PFKFB3 expression was positively correlated with AGPG expression. (Pearson's correlation analysis, $n=10$ ). $\mathbf{g}$ In vitro-synthesized $\mathrm{FL}$ and truncation mutants of $A G P G$ were incubated with protein lysates from KYSE150 and KYSE30 cells or with purified His-tagged recombinant PFKFB3. RNA pull-down and western blotting assays were then performed. h CLIP-qPCR showed that the T5 fragment of AGPG was the region responsible for PFKFB3 binding. i RNA pull-down assays showed that AGPG $\triangle T 5$ could not interact with PFKFB3. j AGPG CRISPR KO cell lines were generated using the CRISPR/Cas9 genome-editing system. Overexpression of AGPG FL, but not of AGPG $\triangle T$ 5, was sufficient to reverse the decreased ECAR and cell proliferation caused by AGPG CRISPR KO. $\mathbf{k}$ Western blotting showed that CDK1 downregulation and p27 upregulation by AGPG CRISPR KO were abolished by AGPG FL but not by AGPG $\triangle$ T5. I HomeR was used to perform the motif analysis on the binding peaks obtained by the Piranha and CIMS analyses. Both methods suggested that CCAGCCA might be responsible for PFKFB3 binding. Data in $\mathbf{c}, \mathbf{f}, \mathbf{h}, \mathbf{j}$ are representative of three independent experiments and presented as mean \pm S.D., $n=3$ biologically independent samples, the $P$ value was determined by a two-tailed unpaired Student's $t$ test.

transcriptional activity of reporters with an intact p53-BR (Fig. 5h, Supplementary Fig. 5d). Collectively, our data demonstrate the important regulatory role of p53 in AGPG transcription, and loss or mutation of TP53 leads to the striking upregulation of AGPG.

Multiple microenvironmental factors, including hypoxia, DNA damage, and oncogene expression, can affect TP53 status ${ }^{26}$, so we investigated whether these factors are involved in the $A G P G$ regulatory network (Fig. 5i). Expression of oncogenic KRas ${ }^{G 12 \mathrm{~V}}$ led to AGPG upregulation and TP53 downregulation in 293 $\mathrm{T}$ cells ${ }^{27}$ (Fig. $5 \mathrm{j}$ ), indicating that oncogene stress is involved in $A G P G$ regulation by affecting TP53 status.

We also extended our analysis to hypoxic conditions by detecting AGPG and TP53 expression in cells exposed to severe hypoxia or normoxia for $48 \mathrm{~h}$. Both WT and MT p53 were upregulated as previously reported; $A G P G$ expression was decreased in TP53 WT cells (KYSE150) and was markedly increased in TP53 MT cells (TE-1 and KYSE30) after exposure to hypoxia (Fig. 5k). These results suggest that hypoxia-induced $A G P G$ upregulation could be abolished in the presence of WT TP53.

Effects of $A G P G$ on ESCC tumor growth in vivo. Then, we explored the role of $A G P G$ in tumorigenesis in vivo. AGPG knockdown significantly repressed cell-based xenograft tumor growth (Fig. 6a-c, Supplementary Fig. 6a, b). As indicated by the Haemotoxylin and Eosin (HE) and immunohistochemistry (IHC) results, AGPG knockdown decreased the levels of the cell proliferation marker Ki67, as well as of PFKFB3 and CDK1, but increased p27 levels (Fig. 6d, e, Supplementary Fig. 6c, d); these results are consistent with our in vitro experimental results.

Furthermore, in the patient-derived xenograft (PDX) models (generated using tumor tissues from two ESCC patients, SYSUCC) (Fig. 6f), AGPG depletion via in vivo-optimized AGPG inhibitor dramatically reduced tumor growth (Fig. $6 \mathrm{~g}-\mathrm{i}$, Supplementary Fig. $6 \mathrm{e}-\mathrm{g}$ ), implicating $A G P G$ as a promising therapeutic target. The main component of the in vivo-optimized $A G P G$ inhibitor used in the PDX model was antisense oligonucleotides, which exhibited a stronger knockdown effect on nuclear-localized $\mathrm{RNAs}^{28}$. Accordingly, as shown in the HE and IHC analyses, AGPG knockdown significantly affected cell proliferation (indicated by Ki67), which might be attributed to the modulation of PFKFB3 and downstream CDK1 and p27 expression, as mentioned previously (Fig. $6 \mathrm{j}-\mathrm{n}$ ).

The p53-AGPG-PFKFB3 axis is involved in ESCC development. To establish whether the p53-AGPG-PFKFB3 axis is clinically associated and pathologically involved in ESCC development, we detected $A G P G$ expression by qPCR and Ki67, PFKFB3, CDK1, p27, and p53 expression by IHC in a cohort of ESCC tissues (SYSUCC, $n=102$ ). The AGPG-high group exhibited higher Ki67, PFKFB3, and CDK1 expression but lower p27 and p53 expression, whereas the AGPG-low group showed the opposite pattern (Fig. 7a, b). Collectively, we speculated that dysregulation of the p53-AGPG-PFKFB3 axis promotes ESCC development.

In addition, we examined PFKFB3 expression in a set of ESCC and matched normal tissues by IHC. In agreement with previous reports, PFKFB3 was highly expressed in malignant tissues ${ }^{29-31}$ (Fig. 7c, d), and high PFKFB3 expression was associated with poor outcomes in ESCC patients (Fig. 7e, SYSUCC, $n=104$; clinicopathological information is provided in Supplementary Table 4). We next examined AGPG expression in these tissues by RNAScope ISH assays. Then, the tissues were sorted into the AGPG/PFKFB3-high, AGPG/PFKFB3-intermediate, and AGPG/ PFKFB3-low groups, among which the AGPG/PFKFB3-high subset had a much worse prognosis than the other subsets (Fig. 7f, SYSUCC, $n=104$; clinicopathological information is provided in Supplementary Table 5). These data further indicate $A G P G / P F K F B 3$ as a promising prognostic indicator and a potential therapeutic target.

\section{Discussion}

Cancer cells reprogram glucose metabolism toward aerobic glycolysis to increase their biomass and sustain uncontrolled proliferation ${ }^{32}$. Apart from its well-recognized role in glycolysis regulation, PFKFB3 has been shown in many studies to have an important role in cell cycle progression, in which it catalyzes the production of F-2,6-BP, which functions as a regulator of CDK1 and p27. Thus, PFKFB3 has critical roles in cancer cells by linking glycolysis to cell proliferation. PFKFB3 levels are regulated both transcriptionally and posttranslationally. PFKFB3 is reported to be dimethylated at R131 and R134, and the regulation of PFKFB3 methylation determines directional glucose utilization ${ }^{17}$. The acetylation of PFKFB3 induced by cisplatin impairs PFKFB3 translocation to the nucleus and causes PFKFB3 accumulation in the cytoplasm, leading to increased glycolysis and protecting against DNA damage ${ }^{33}$. Collectively, posttranslational modifications determine the stability and activity of PFKFB3.

At present, the list of lncRNAs involved in tumor progression is rapidly expanding. A few lncRNAs have been implicated in cancer metabolism regulation, but the underlying mechanisms remain poorly understood. Here, we found that the lncRNA AGPG significantly influences cell proliferation by directly binding to and stabilizing the key enzyme PFKFB3, which regulates both glucose metabolism and the cell cycle. Canonical RNA-binding proteins 
a

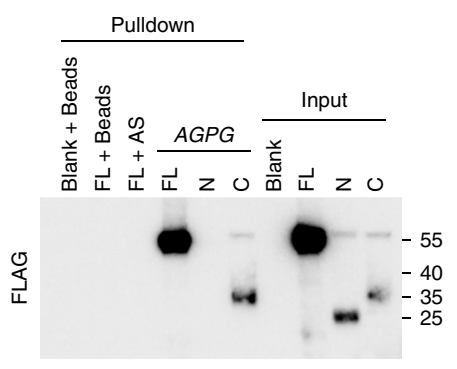

b

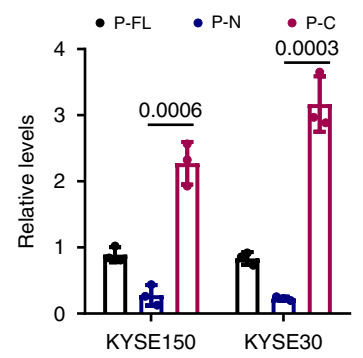

C

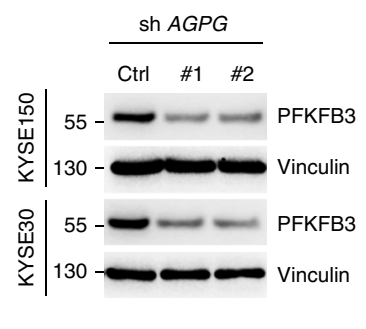

d

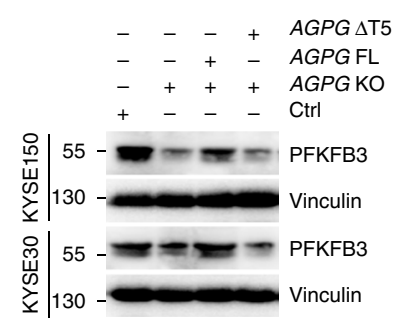

e

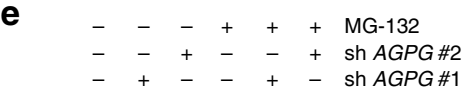

$-+\ldots++-\operatorname{sh} A G P G \# 1$

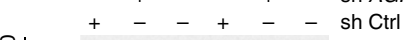

点 $55-\longrightarrow$ PFKFB3

岁| $130-\infty-\infty-\infty-2$ Vinculin

兽 55 - PFKFB3

Vinculin f

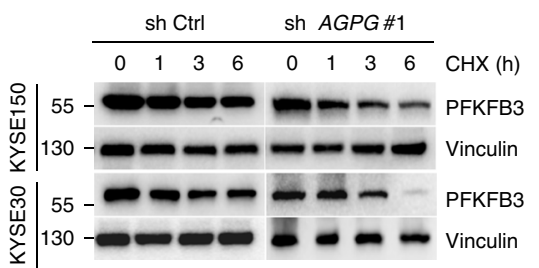

g

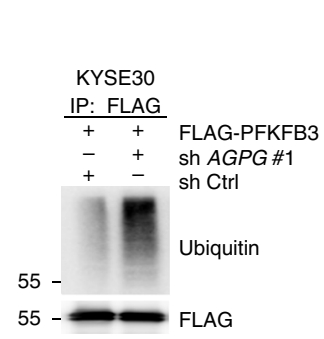

h

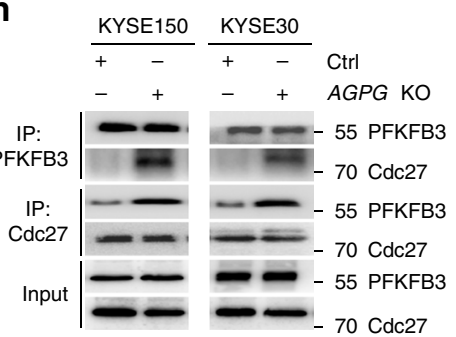

i

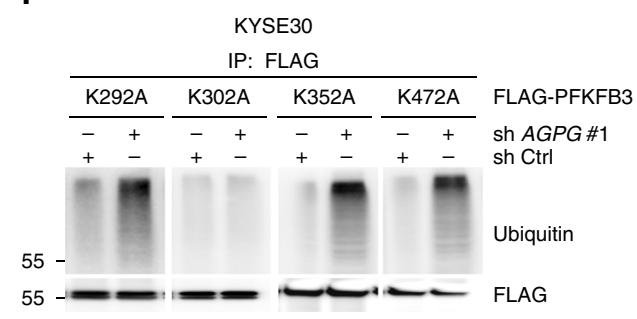

j

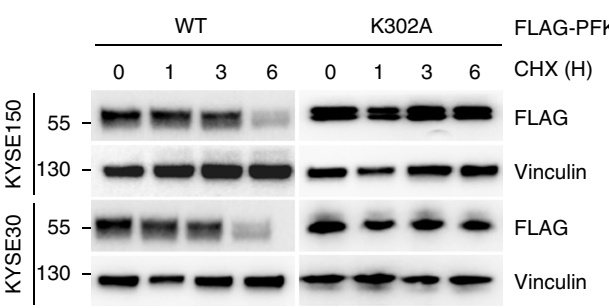

k
KYSE150 $\rightarrow$ Ctrl + Vector
$\rightarrow$ AGPG CRISPR KO + Vector $\rightarrow$ AGPG CRISPR KO + PFKFB3 WT

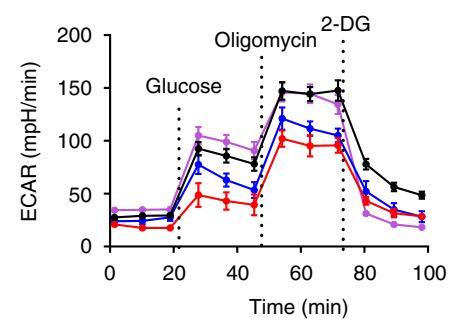

\section{I}

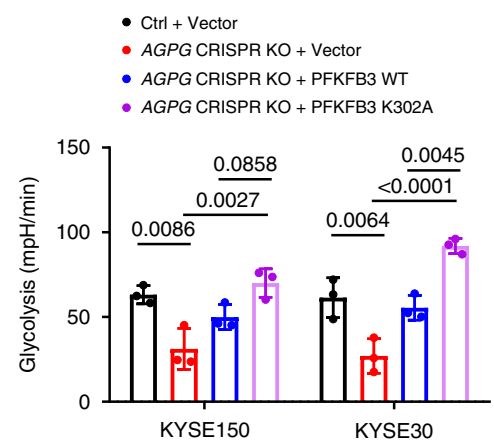

m
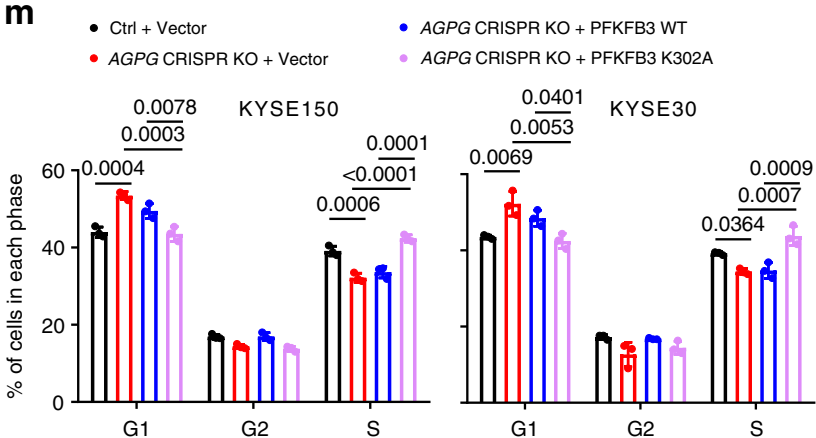

(RBPs) generally contain RNA-binding regions, but recent studies have indicated that hundreds of new RBPs lack known RNA-binding domains, indicating the complexity and diversity of RNA-protein complexes ${ }^{34,35}$. A large number of RBPs are gradually being discovered using various experimental methods. In our study, we performed a variety of in vitro and in vivo experiments to study the interaction between the IncRNA and the protein, including RNA pull-down, RIP, CLIP, and MTRAP assays. Consistently, we demonstrated that $A G P G$ is directly associated with PFKFB3, which implies that PFKFB3 is a newly discovered noncanonical RBP.

The mechanism of PFKFB3 stabilization remains largely unknown. Recent studies have shown that polyubiquitination of PFKFB3 is a critical step leading to degradation through the E3 
Fig. 4 AGPG affects PFKFB3 stability by preventing its ubiquitination. a In vitro-synthesized AGPG was incubated with protein lysates from KYSE30 cells transfected with vectors expressing FLAG-tagged FL or truncation mutants of PFKFB3. RNA pull-down and western blotting assays were then performed. Truncation mutants included FL, N-terminal (N) and C-terminal (C) constructs. b RIP assays were performed using anti-FLAG antibodies in cells transfected with vectors expressing FLAG-tagged FL or truncation mutants of PFKFB3. c AGPG knockdown reduced PFKFB3 expression in ESCC cells. d PFKFB3 downregulation by AGPG CRISPR KO was rescued by AGPG FL but not by AGPG $\triangle T 5$. e PFKFB3 downregulation by AGPG knockdown was abolished by MG-132 (10 $\mu \mathrm{M}, 12 \mathrm{~h})$. f Western blotting detection of PFKFB3 levels in KYSE150 cells transfected with shCtrl or shAGPG followed by treatment with $\mathrm{CHX}(100 \mu \mathrm{g}$ per $\mathrm{ml})$ for the indicated times. $\mathbf{g}$ IP assays showed that AGPG knockdown increased PFKFB3 ubiquitination levels. FLAGtagged PFKFB3 was expressed in cells, which were then subjected to IP assays. $\mathbf{h}$ Active APC/C could be immunoprecipitated from cells using monoclonal Cdc27 antibody. ColP assays showed that AGPG CRISPR KO significantly increased the interaction between PFKFB3 and Cdc27. i IP assays showed that AGPG knockdown did not increase ubiquitination of the PFKFB3 K302A mutant. $\mathbf{j}$ Cells were infected with FLAG-tagged PFKFB3 WT or K302A and treated with $\mathrm{CHX}(100 \mu \mathrm{g}$ per $\mathrm{ml})$ for the indicated time. FLAG levels were detected by western blotting. $\mathbf{k}$ PFKFB3 K302A overexpression significantly reversed the decreased ECAR and cell proliferation caused by AGPG CRISPR KO, whereas PFKFB3 WT could only partially rescue these effects in KYSE150 cells. I PFKFB3 K302A overexpression significantly reversed the decreased glycolysis caused by AGPG CRISPR KO, whereas PFKFB3 WT could only partially rescue this effect. $\mathbf{m}$ PFKFB3 K302A overexpression abolished the G1/S arrest caused by AGPG CRISPR KO, whereas PFKFB3 WT could only partially rescue this effect. Data in $\mathbf{b}, \mathbf{k}-\mathbf{m}$ are representative of three independent experiments and presented as mean \pm S.D., $n=3$ biologically independent samples, the $P$ value was determined by one-way ANOVA with Tukey's multiple comparisons test. No adjustments were made for multiple comparisons.

ubiquitin ligase APC/C-Cdh1. Our study suggests that AGPG specifically binds to $\mathrm{PFKFB} 3$ and blocks its interaction with APC/C, which inhibits APC/C-mediated PFKFB3 ubiquitination and subsequent degradation. As reported, PFKFB3 is subject to constant polyubiquitination at several sites, including K142, and proteasomal degradation ${ }^{21}$. Our study also showed that K302 is an important ubiquitination site responsible for PFKFB3 stability, and upon interacting with PFKFB3, AGPG blocks K302 ubiquitination and enhances PFKFB3 stability. Collectively, these findings further delineate the detailed mechanism underlying lncRNA-mediated PFKFB3 turnover and cancer metabolism remodeling.

Considering the oncogenic role of $A G P G$, we also investigated the mechanism of $A G P G$ regulation, which was associated with the p53 pathway. Cells exposed to severe hypoxia, nutrient deprivation, or genotoxic insults in the tumor microenvironment are characterized by the stabilization and activation of $\mathrm{p} 53^{26,36}$. Studies have revealed that many lncRNAs are transcriptional targets of p53 $25,37,38$. However, the exact p53 target genes responsible for glucose metabolism remain poorly characterized ${ }^{39,40}$. p53 can directly repress transcription by binding p53 response elements ${ }^{41,42}$, and this interaction may represent a key regulatory link in the p53-mediated cellular response. In this study, we found that $A G P G$ is a transcriptional target negatively regulated by $\mathrm{p} 53$ through the identified $\mathrm{p} 53-\mathrm{BR}$ in the $A G P G$ promoter. These data further verified the tumor suppressive function of $\mathrm{p} 53$ in regulating metabolism. Unfortunately, over $50 \%$ of human cancers, including ESCC, have no WT TP53 function due to mutation or deletion $^{43,44}$, and multiple microenvironmental factors, including hypoxia and excessive genotoxic insults, are potential driving forces that cause TP53 mutations ${ }^{45}$. Therefore, dysregulation of the p53-AGPG-PFKFB3 axis leads to metabolism remodeling and cell proliferation.

As a predominant histologic type of malignant esophageal tumor, ESCC is prevalent worldwide, especially in certain regions ${ }^{46-48}$. Although multiple therapies, including surgery, radiotherapy, and chemotherapy, have been used, ESCC remains a leading cause of cancer-related death ${ }^{49}$. Even worse, current studies on targeted therapeutic approaches or biomarker-driven therapies for this malignant disease are not promising. Therefore, it is important to elucidate the molecular mechanisms underlying ESCC and to develop more effective therapies. Our study showed that both AGPG and PFKFB3 are highly expressed in ESCC and that high expression of either $A G P G$ or PFKFB3 is closely linked to unfavorable outcomes. High expression of both $A G P G$ and PFKFB3 is correlated with an even poorer prognosis, suggesting that the combination of $A G P G$ and PFKFB3 is a potential prognostic marker for ESCC diagnosis. In addition to ESCC, $A G P G$ is also highly expressed in multiple types of cancer, suggesting that $A G P G$ might be a promising pancancer therapeutic target.

In conclusion, our study showed that $A G P G$, a transcriptional target of p53, has a pivotal role in promoting glycolysis and cell proliferation by enhancing PFKFB3 stability, thus facilitating the development of cancer. Our findings provide a basis for RNA interference-based strategies that target lncRNAs and cancer metabolism for cancer treatment.

\section{Methods}

Cell lines. Het-1A, NE-1, and HCT-116 cells were obtained from American Type Culture Collection (ATCC, Rockville, MD, USA). KYSE30, KYSE510, KYSE150, KYSE520, KYSE70, and KYSE180 cells were obtained from German Cell Culture Collection (DSMZ, Braunschweig, Germany). TE-1, TE-9, and TE-15 cells were obtained from the Cell Bank of Shanghai Institute of Cell Biology (Chinese Academy of Medical Sciences, Shanghai, China). HCT-116 (TP53 KO) and 293 T inducible KRas ${ }^{G 12 V}$ (iK-Ras ${ }^{G 12 V}$ ) cells were provided by professor Peng Huang (SYSUCC, Guangzhou, China). The cells were maintained in RPMI-1640 (HyClone, Logan, UT, USA) supplemented with $10 \%$ fetal bovine serum (Invitrogen, Carlsbad, CA, USA) and $1 \%$ penicillin/streptomycin (HyClone) at $37^{\circ} \mathrm{C}$ with $5 \% \mathrm{CO}_{2}$. Based on short tandem repeat (STR) profiling by vendors, no cells used in this study are found in the database of commonly misidentified cell lines. All cells were further authenticated via STR-PCR DNA profiling by Guangzhou Cellcook Biotech Co.,Ltd. (Guangzhou, China) and were determined to be free of mycoplasma contamination.

Human tissue specimens. Clinical samples were collected from SYSUCC (Guangzhou, China). All patients had a histological diagnosis of cancer. After the operation, the patients received regular follow-up. All clinicopathological information is provided in Supplementary Tables 1, 4, and 5.

Reagents, plasmid construction, and site-directed mutagenesis. MG-132 was purchased from Selleck Chemicals (Houston, TX, USA). Cycloheximide (CHX) and doxycycline were purchased from Sigma-Aldrich (St. Louis, MO, USA). Recombinant human PFKFB3 protein with an N-terminal His tag was purchased from Novus Biologicals (Littleton, Colorado). N-Terminal FLAG-tagged expression vectors (for expression in mammalian cells) for FL PFKFB3, truncated mutants, and site-directed mutants (K292A, K302A, K352A, and K472A) were provided by OBiO Technology (Shanghai, China). Expression vectors for FL AGPG and truncated mutants used for in vitro RNA synthesis were provided by OBiO Technology (Shanghai, China). p53 and Cdc27 expression vectors were provided by GeneCopoeia Inc. (Rockville, MD, USA).

RNA interference. Cell transfections and lentiviral transductions were performed according to the manufacturer's instructions ${ }^{7}$. Small interfering RNAs (siRNAs) and short hairpin RNAs (shRNAs) were provided by RiboBio (Guangzhou, China) or OBiO Technology (Shanghai, China). The resulting constructs were verified by sequencing. The sequences are listed in Supplementary Table 6.

CRISPR/Cas9-mediated gene editing. AGPG KO cell lines were generated using a CRISPR/Cas9-based strategy ${ }^{50}$. AGPG-specific guide RNA (gRNA) expression 
a

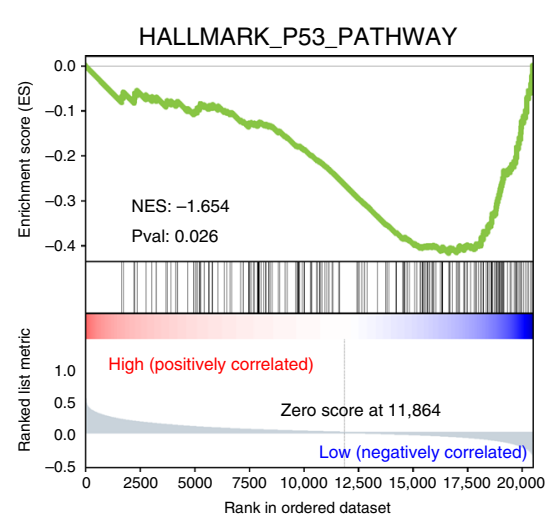

e

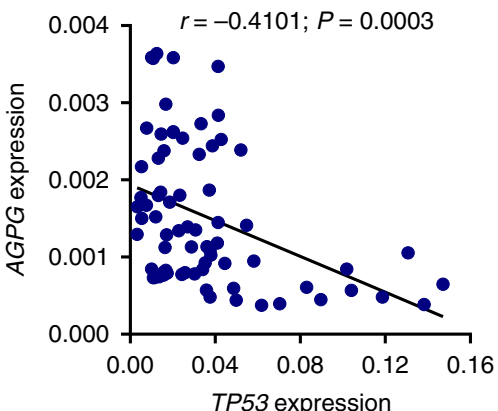

i

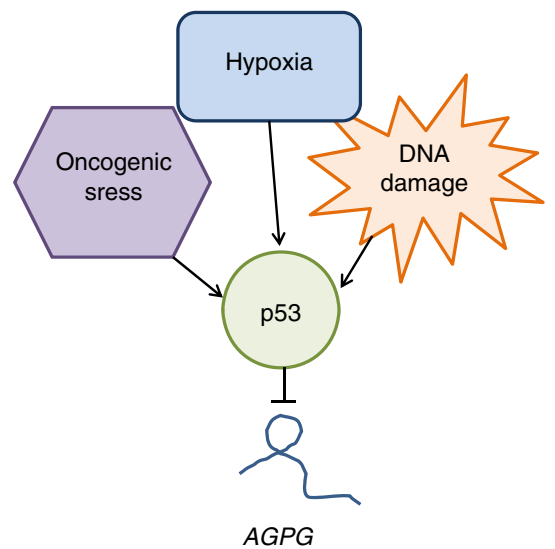

b

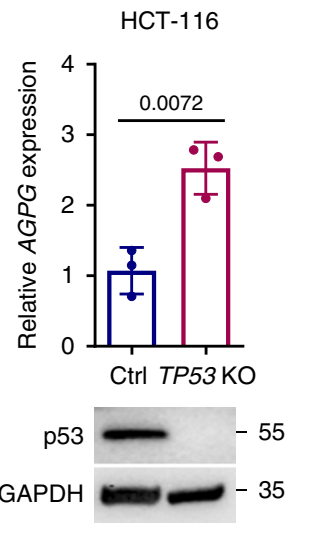

f

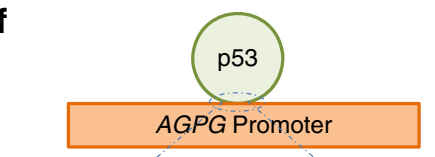

922 CGCTGGCCAGTCAAGTT 939

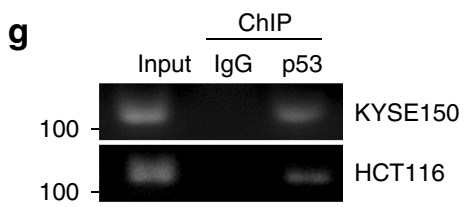

j

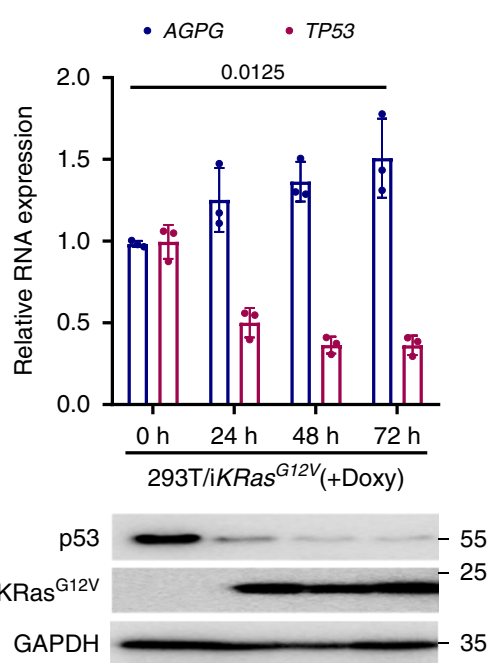

d $\cdot$ Ctrl

- TP53 WT overexpression

- TP53 Knockdown
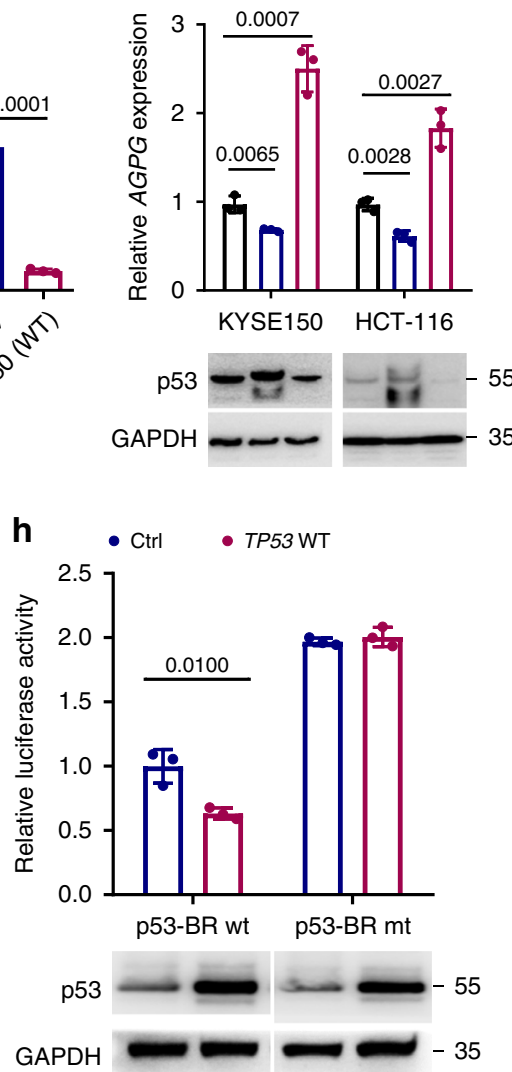

K - Normoxia - Hypoxia
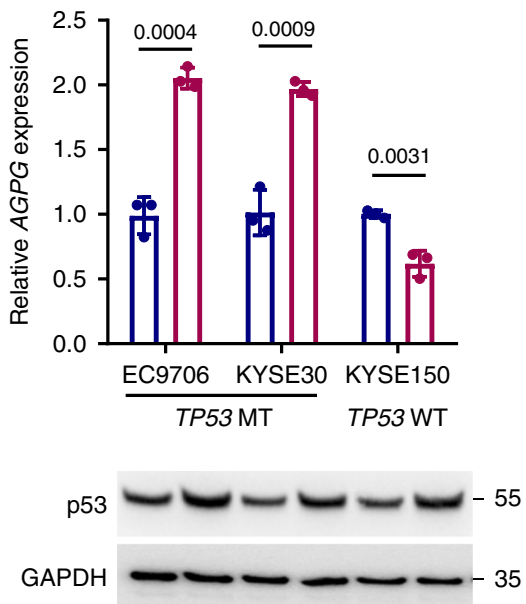

Fig. 5 AGPG is transcriptionally regulated by p53. a Pathway enrichment analysis suggested that AGPG was negatively correlated with p53. The pathway analysis was performed with GSEA method, which was based on an empirical permutation test procedure. $\mathbf{b}$ qPCR detection of AGPG expression in TP53 $\mathrm{KO}$ and control HCT-116 cells. c qPCR detection of AGPG expression in ESCC cells with WT TP53 (KYSE150) or MT TP53 (KYSE30 and TE-1). d qPCR detection of AGPG expression in KYSE150 and HCT-116 cells with p53 upregulation or downregulation or control. e qPCR analysis showed that AGPG expression was negatively correlated with TP53 expression in a cohort of ESCC patients with WT TP53 (SYSUCC, Pearson's correlation analysis, $n=72$ ). f The AGPG promoter contains a consensus p53-binding region. $\mathbf{g}$ ChIP assays showed that p53 bound to the AGPG promoter. $\mathbf{h}$ WT TP53 overexpression diminished the transcriptional activity of AGPG in KYSE150 cells. i Schematic map of the regulatory network involving p53 and AGPG. $\mathbf{j}$ qPCR detection of AGPG expression in 293 T cells exposed to oncogenic stress (20 ng per ml doxycycline to induce ectopic KRas G12V expression) for 0, 24, 48, or $72 \mathrm{~h}$. Doxy, doxycycline. $\mathbf{k}$ qPCR detection of AGPG expression in cells exposed to hypoxia or normoxia for $48 \mathrm{~h}$. Data in $\mathbf{b}-\mathbf{d}, \mathbf{h}, \mathbf{j}, \mathbf{k}$ are representative of three independent experiments and presented as mean \pm S.D., $n=3$ biologically independent samples, the $P$ value was determined by a two-tailed unpaired Student's $t$ test. 
a
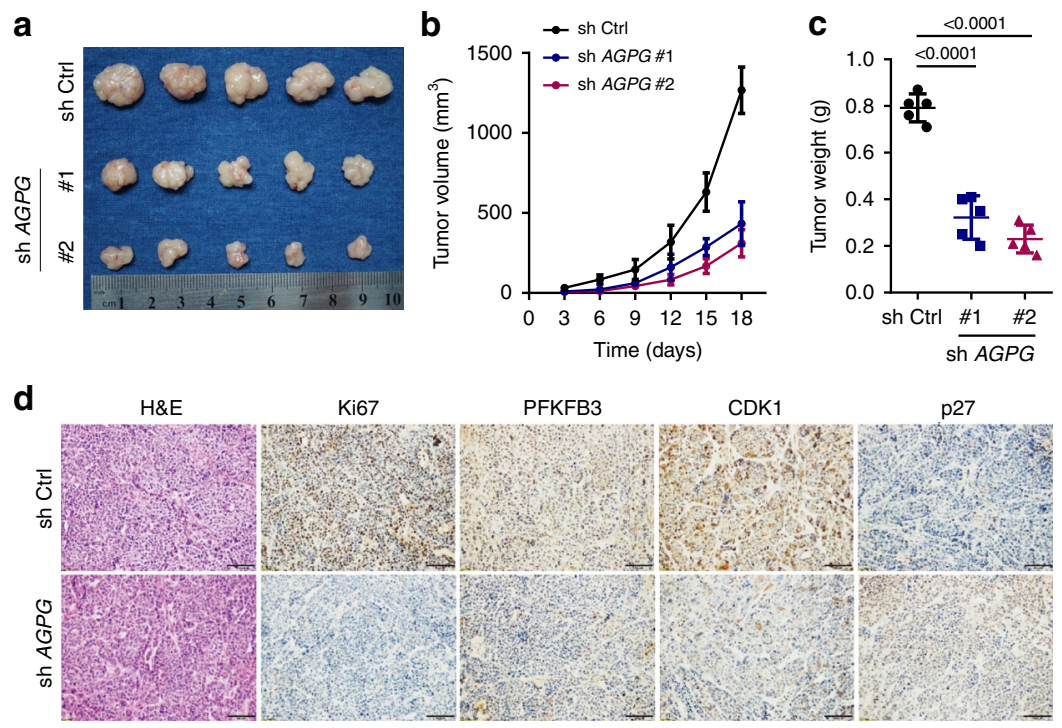

f
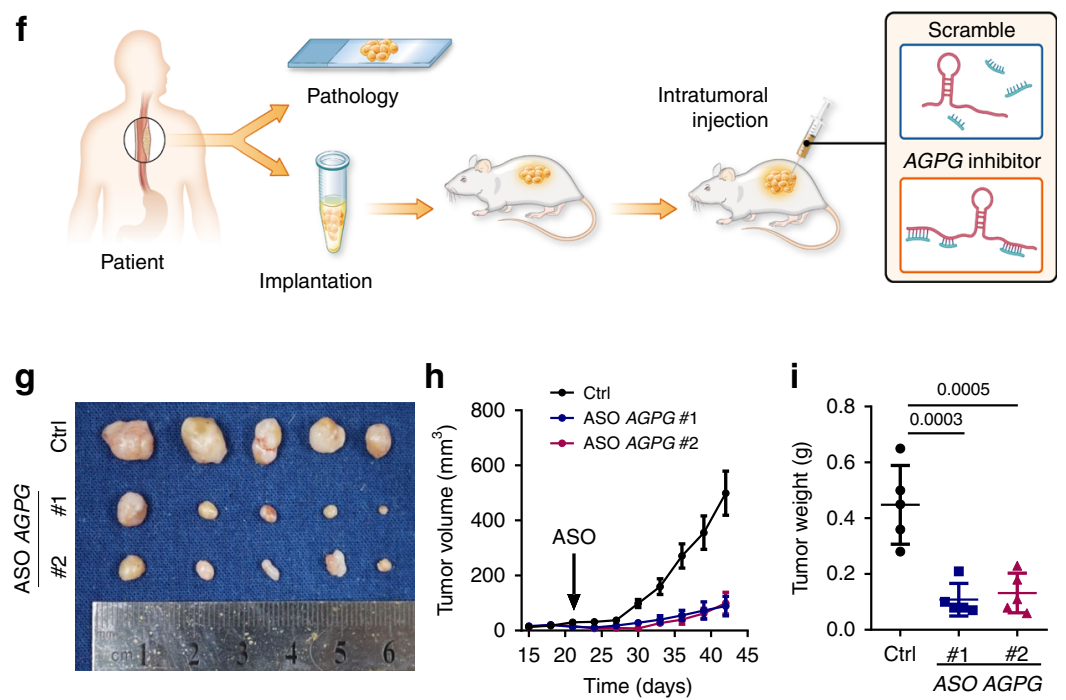

j

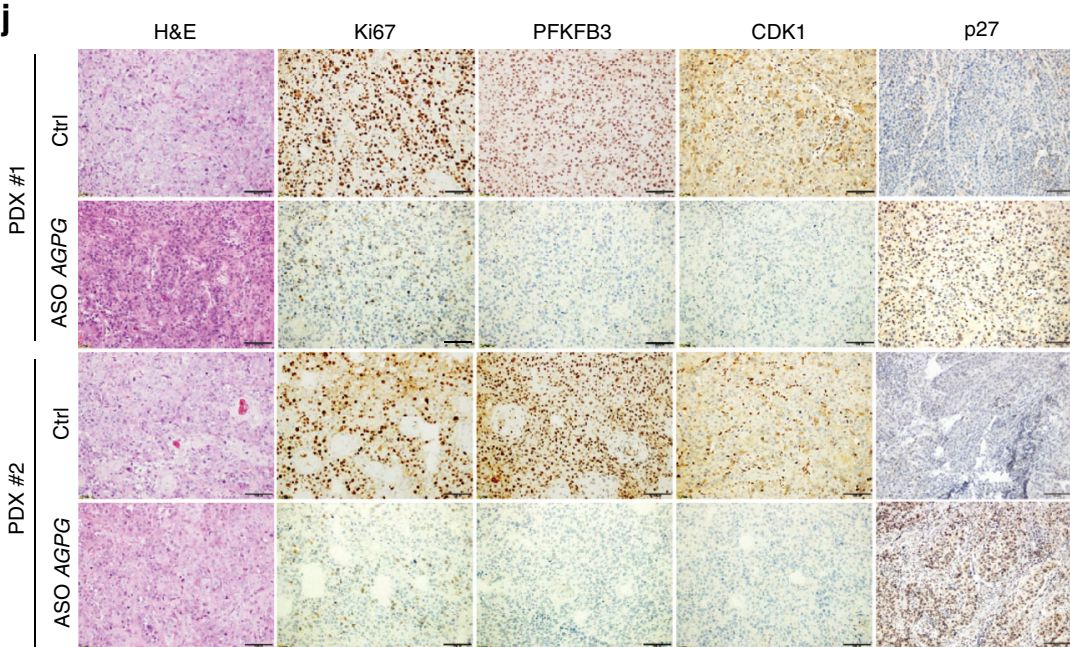

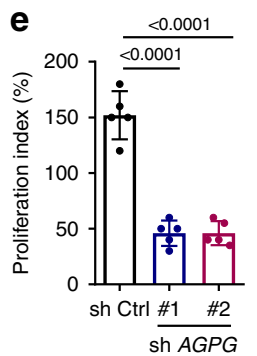
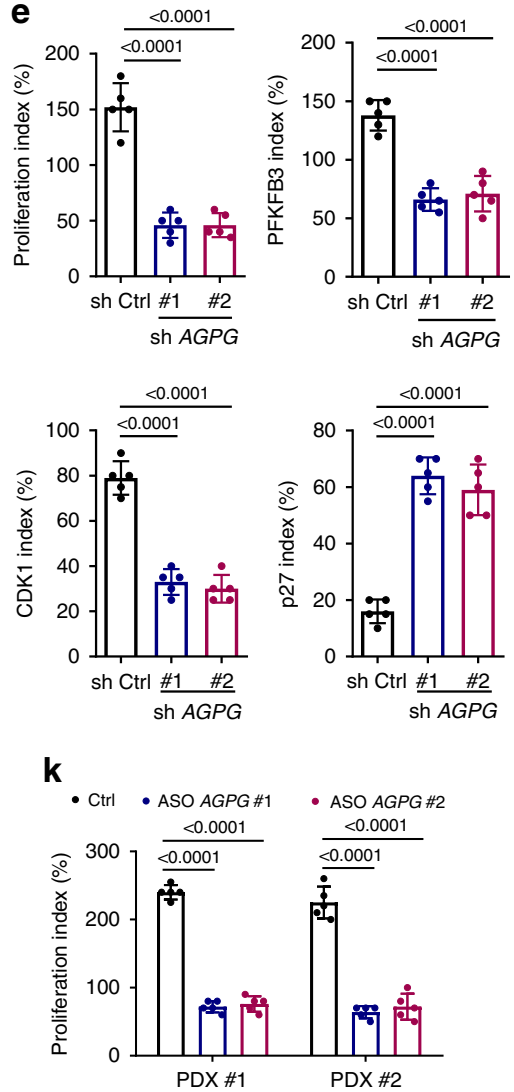

I

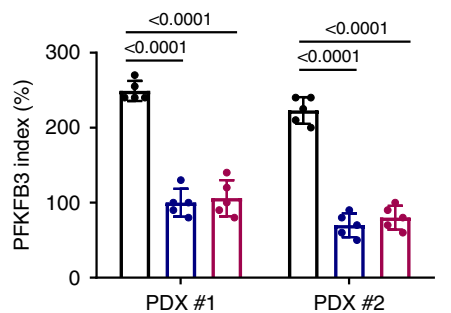

m
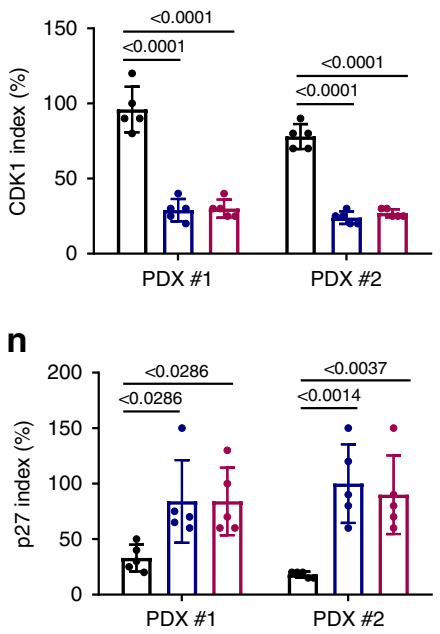

vectors were obtained from Kidan Biotechnology Co., Ltd. (Guangzhou, China). Briefly, AGPG-specific sgRNAs were designed to recognize two different sites of the AGPG gene, the location of the upstream target (>hg38_refGene_NR_002929_0 range $=$ chr1:202861697-202861719) and the location of the downstream target ( $>$ hg38_refGene NR 002929_6 range $=$ chr1:202876356-202876378). To identify the effective sgRNAs, we transfected different sgRNA-Cas9 vectors into $293 \mathrm{H}$ cells, and then, the genomic DNA was extracted, Q5 PCR was performed and the PCR products were subjected to sequencing. The results showed that AGPG sgRNA01 (CGGCGGGGCTGTTTCGTAAG) was effective among the upstream sgRNAs, and AGPG sgRNA02 (ATCAAGTGTCCTATATGCGT) was effective among the downstream sgRNAs. The PCR primer GCAACACCACGAATCCCAAC/TTGTC CCGCTCTGGAAACTC and the sequencing primer TTGTCCCGCTCTGGAAA

CTC were used to detect the efficiency of $A G P G$ sgRNA01. The PCR primer ACACTAGGCCATGCACCAA/GCCCACAGGCCAAATTCATTC and the 
Fig. 6 Effects of AGPG on tumor growth in vivo. a AGPG knockdown inhibited cell-based xenograft growth in nude mice. b, c Statistical analysis of KYSE150 tumor volume and weight in nude mice. d Representative IHC images of randomly selected KYSE150 cell-based tumors from each group. Scale bar, $100 \mu \mathrm{m}$. e Quantification of IHC staining in KYSE150 cell-based tumors. f Graphic illustration of the intratumoral injection of in vivo-optimized AGPG inhibitor or control in the PDX models. $\mathbf{g}$ AGPG knockdown inhibited PDX growth in nude mice. The tumor tissues were from two ESCC patients (SYSUCC). $\mathbf{h}$, i Statistical analysis of tumor volume and weight in the PDX \#1 model. $\mathbf{j}$ Representative IHC images of randomly selected human-derived tumors from each group. Scale bar, $100 \mu \mathrm{m}$. k-n Quantification of IHC staining in human-derived tumors. Data in $\mathbf{b}, \mathbf{c}, \mathbf{e}, \mathbf{h}, \mathbf{i}, \mathbf{k}-\mathbf{n}$ are representative of three independent experiments and presented as mean \pm S.D., $n=5$ mice per group, the $P$ value was determined by one-way ANOVA with Dunnett's multiple comparisons test. No adjustments were made for multiple comparisons.

sequencing primer GCCTCAGCCCACAGAGCTTA were used to detect the efficiency of $A G P G$ sgRNA02. Thus, double sgRNA vectors were constructed using the sgRNAs mentioned above. After the construction of the vectors, the vectors were sequenced and compared with the target genes, which showed that the vectors were constructed correctly. ESCC cells were transfected with the pLV-U6-AGPG RNA sgRNA01-7SK-sgRNA02-EFS-hCas9-2A-Puro or pLV-U6-NC sgRNA01-7SK-NC sgRNA02-EFS-hCas9-2A-Puro expression vectors. The transfected cells were selected using puromycin $(1 \mu \mathrm{g}$ per $\mathrm{ml})$ for 7 days. Isolated single colonies were expanded and subjected to detection of genomic deletions by PCR.

Cell proliferation and cell cycle analysis. Cell proliferation was measured with MTS (Qiagen, Hilden, German) according to the manufacturer's instructions ${ }^{25}$. Cell cycle analysis was performed with a cell cycle detection kit (KeyGen, China) according to the manufacturer's instructions. The cells were then analyzed with a Gallio flow cytometer (Beckman Coulter, CA, USA) and MultiCycle for Windows (Phoenix Flow Systems, CA, USA). The gating strategy for cell cycle analysis was provided in Supplementary Fig. 7

Western blot and qPCR analysis. Western blot analysis was performed according to the manufacturer's instructions 7 . Cells or tissues were lysed in RIPA buffer. The protein concentrations were normalized with a BCA assay kit (Thermo Fisher Scientific, Carlsbad, CA, USA). Anti-GAPDH (1:1000, Cell Signaling Technology, Beverly, USA, 5174), anti-vinculin (1:1000, Cell Signaling Technology, 13901), anti-PFKFB3 (1:1000, Abcam, Cambridge, MA, USA, ab181861), anti-PFKFB3 (phospho S461)(1:1000, ab232498), anti-p27 (1:1000, Abcam, ab32034), antiCDK1 (1:1000, Abcam, ab133327), anti-p53 (1:1000, Abcam, ab1101), anti-p21 (1:1000, Abcam, ab109199), anti-CDK3 (1:1000, Abcam, ab96847), anti-CDK6 (1:1000, Abcam, ab124821), anti-FLAG tag (1:1000, Cell Signaling Technology, 8146), anti-His tag (1:1000, Abcam, ab9108), anti-Cdc27 (1:1000, Abcam, ab10538), and anti-ubiquitin antibodies (1:1000, Cell Signaling Technology, 3933) were used in this study. Unprocessed images of the immunoblots are provided in the Supplementary Fig. 8. RNA levels were measured by qPCR analysis according to the manufacturer's instructions ${ }^{51}$. The specific primer sequences are listed in Supplementary Table 7 .

Determination of glycolytic activity. Media samples were collected from cells cultured for $12 \mathrm{~h}$ or $24 \mathrm{~h}$. Lactate concentrations were determined by a biosensor. ECAR was detected according to the XF Glycolysis Stress Test protocol on a Seahorse XFe24 Extracellular Flux Analyzer (Agilent Technologies, Santa Clara, CA, USA). ${ }^{13} \mathrm{C}$-Labeled intracellular metabolites were detected as previously described ${ }^{17}$. Cells $\left(2 \times 10^{7}\right)$ were incubated with 2 g per $\mathrm{L}^{13} \mathrm{C}_{6}$-labeled glucose (Cambridge Isotope Laboratories, CLM-1396-10) for $2 \mathrm{~h}$. Metabolites were extracted, and those including at least one ${ }^{13} \mathrm{C}$ atom were analyzed using an LC system equipped with a TripleTOF 5600 mass spectrometer (SCIEX, Framingham, MA, USA). The concentrations of lactate, ECAR, and ${ }^{13} \mathrm{C}_{6}$-labeled metabolites were normalized to cell number.

RNA pull-down and RIP assays. RNA was transcribed in vitro using a MEGAscript T7 Transcription Kit (Invitrogen) and biotinylated using a Pierce RNA 3' End Desthiobiotinylation Kit (Thermo Scientific) according to the manufacturers' instructions. Cells were prepared using Pierce IP lysis buffer (Thermo Scientific). RNA pull-down assays were performed with a Pierce Magnetic RNA-Protein PullDown Kit. According to the manufacturer's instructions, biotinylated RNA was captured with streptavidin magnetic beads and then incubated with cell lysates or purified protein $(20 \mu \mathrm{g})$ at $4{ }^{\circ} \mathrm{C}$ for $6 \mathrm{~h}$ before washing and elution of the RBP complex. The eluted proteins were subjected to MS analysis or western blotting. RIP assays were performed with a Magna RNA-binding protein immunoprecipitation kit (Millipore, Bedford, MA) according to the manufacturer's instructions. Negative control IgG, human anti-PFKFB3 antibody (1:20, Abcam, ab181861) and anti-FLAG tag antibody (1:20, Cell Signaling Technology, 8146) were used in this study. After proteinase K digestion, the immunoprecipitated RNAs were extracted, purified, and subjected to qPCR. RNA levels were normalized to the input (10\%).

MS2-tagged RNA affinity purification. ESCC cells were cotransfected with pcDNA3.1-MS2/pcDNA3.1-MS2-AGPG and MCP-3xFLAG plasmids (OBiO
Technology, Shanghai, China). After 48 h, living cells were irradiated with $254 \mathrm{~nm}$ UV light at $400 \mathrm{~mJ}$ per $\mathrm{cm}^{2}$. Then, the cells were lysed for $10 \mathrm{~min}$ on ice and centrifuged at 13,000 $\times g$ for $10 \mathrm{~min}$. FLAG-tagged proteins were immunoprecipitated with anti-FLAG M2 affinity gel (Sigma-Aldrich, USA). After three washes with low-salt wash buffer, agarose gels were boiled in loading buffer, and proteins were detected by western blotting analysis.

CLIP-qPCR. For sixteen hours before UVA exposure, 4-thiouridine (4-SU) was added to the ESCC cell culture medium at a final concentration of $100 \mu \mathrm{m}$. Then, the living cells were irradiated with $150 \mathrm{~mJ}$ per $\mathrm{cm}^{2}$ UVA $(365 \mathrm{~nm})$ and lysed with NP-40 lysis buffer supplemented with protease inhibitors and $1 \mathrm{~mm}$ DTT. RNase $\mathrm{T} 1$ was added to the supernatant at a final concentration of $1 \mathrm{U}$ per $\mu \mathrm{l}$, and the samples were incubated at $22^{\circ} \mathrm{C}$ for $15 \mathrm{~min}$. Then, $40 \mu \mathrm{l}$ of bead slurry was incubated with $10 \mu$ g of normal IgG or PFKFB3 antibody (Abcam, ab181861) for $2 \mathrm{~h}$ at $4^{\circ} \mathrm{C}$ in NT2 buffer. The beads were washed, $1 \mathrm{~mL}$ of cell lysate was added to the antibody-coated Sepharose beads, and the mixtures were incubated for $3 \mathrm{~h}$ at $4{ }^{\circ} \mathrm{C}$. The beads were washed, and the pellets were incubated with 20 units of RNase-free DNase I in $100 \mu \mathrm{l}$ of NP-40 lysis buffer for $15 \mathrm{~min}$ at $37^{\circ} \mathrm{C}$. The pellets were washed and incubated with $0.1 \% \mathrm{SDS}$ and $0.5 \mathrm{mg}$ per ml proteinase $\mathrm{K}$ for $15 \mathrm{~min}$ at $55^{\circ} \mathrm{C}$. Then, the supernatants were collected, RNA was extracted, and qPCR analysis was performed.

HITS-CLIP. Cells were washed with ice-cold PBS three times, and UV crosslinking was performed with UV irradiation type C $(254 \mathrm{~nm})$ at $400 \mathrm{~mJ} \mathrm{per} \mathrm{cm}^{2}$. The crosslinked cells were scraped off the plate and collected by centrifugation at $1000 \times g$ for $5 \mathrm{~min}$. Cell lysis was performed in cold lysis buffer $(1 \times$ PBS, $0.1 \%$ SDS, $0.5 \% \mathrm{NP}-40$ and $0.5 \%$ sodium deoxycholate) supplemented with a $1 \%$ RNase inhibitor (TaKaRa) and $2 \%$ protease inhibitor cocktail (Roche) for $30 \mathrm{~min}$. Cell lysates were cleared by centrifugation at $8000 \times g$ for $10 \mathrm{~min}$ at $4{ }^{\circ} \mathrm{C}$, and the supernatants were used for IP. For DNA digestion, RQ1 (Promega) was added to the lysate and incubated at $37^{\circ} \mathrm{C}$ for $3 \mathrm{~min}$. For IP, $600 \mu \mathrm{L}$ of lysate was incubated with $15 \mu$ g PFKFB3 antibody (Abcam, ab181861) or control IgG antibody overnight at $4{ }^{\circ} \mathrm{C}$. The immunoprecipitates were further incubated with protein $\mathrm{A} / \mathrm{G}$ Dynabeads for $2-3 \mathrm{~h}$ at $4{ }^{\circ} \mathrm{C}$. After the magnet was applied and the supernatant was removed, the beads were sequentially washed twice with wash buffer (1× PBS, $1 \%$ SDS, $0.5 \%$ NP- 40 and $5 \%$ sodium deoxycholate), high-salt wash buffer $(5 \times \mathrm{PBS}, 1 \%$ SDS, $0.5 \%$ NP-40, and 5\% sodium deoxycholate), and PNK buffer (50 mM Tris $\mathrm{pH}=7.4,10 \mathrm{~mm} \mathrm{MgCl}_{2}$, and $\left.0.5 \% \mathrm{NP}-40\right)$. The on-bead digestion was performed by adding MNase (Thermo), followed by incubation at $37^{\circ} \mathrm{C}$ for $10 \mathrm{~min}$. After the samples were washed with PNK buffer as described above, dephosphorylation and phosphorylation were performed with calf intestinal alkaline phosphatase (CIP, NEB) and polynucleotide kinase (PNK, NEB), respectively. The immunoprecipitated protein-RNA complex was eluted from the beads by heat denaturing and was resolved on a Novex $4-12 \%$ Bis-Tris precast polyacrylamide gel (Invitrogen). The protein-RNA complexes were cut from the gel ${ }^{52}$, and RNA was extracted with TRIzol after digesting the proteins. The cDNA libraries were prepared using the KAPA Stranded RNA-Seq Library Preparation Kit (Kapa Biosystems). The cDNAs were purified and amplified, and PCR products corresponding to $200-500 \mathrm{bp}$ were purified, quantified and stored at $-80^{\circ} \mathrm{C}$ before sequencing. For high-throughput sequencing, the libraries were prepared following the manufacturer's instructions and applied to the Illumina HiSeq X Ten system for $150 \mathrm{nt}$ paired-end sequencing by ABlife, Inc. (Wuhan, China). The End $13^{\prime}$ adapter $5^{\prime}$-AGATCGGAAGAGC-3 and the End2 $3^{\prime}$ adaptor $5^{\prime}$-AGATCGGAAGAGC- $3^{\prime}$ were used in this study. We used HomeR to perform the motif analysis on the binding peaks obtained by the Piranha and CIMS analyses ${ }^{19,20}$.

Subcellular fractionation. A Cytoplasmic \& Nuclear RNA Purification Kit (Norgen Biotek Corp, Canada) was used to detect AGPG expression in cytoplasmic and nuclear fractions. According to the manufacturer's instructions, RNA was extracted from the cytoplasmic and nuclear fractions and subjected to qPCR. $\beta$-Actin was used as a cytoplasmic marker, and U6 was used as a nuclear marker.

Fluorescence in situ hybridization (FISH) assay and immunofluorescence staining. FISH assays were carried out with a lncRNA FISH Kit (RiboBio, Guangzhou, China). In brief, cells were fixed and permeabilized in PBS containing 
a
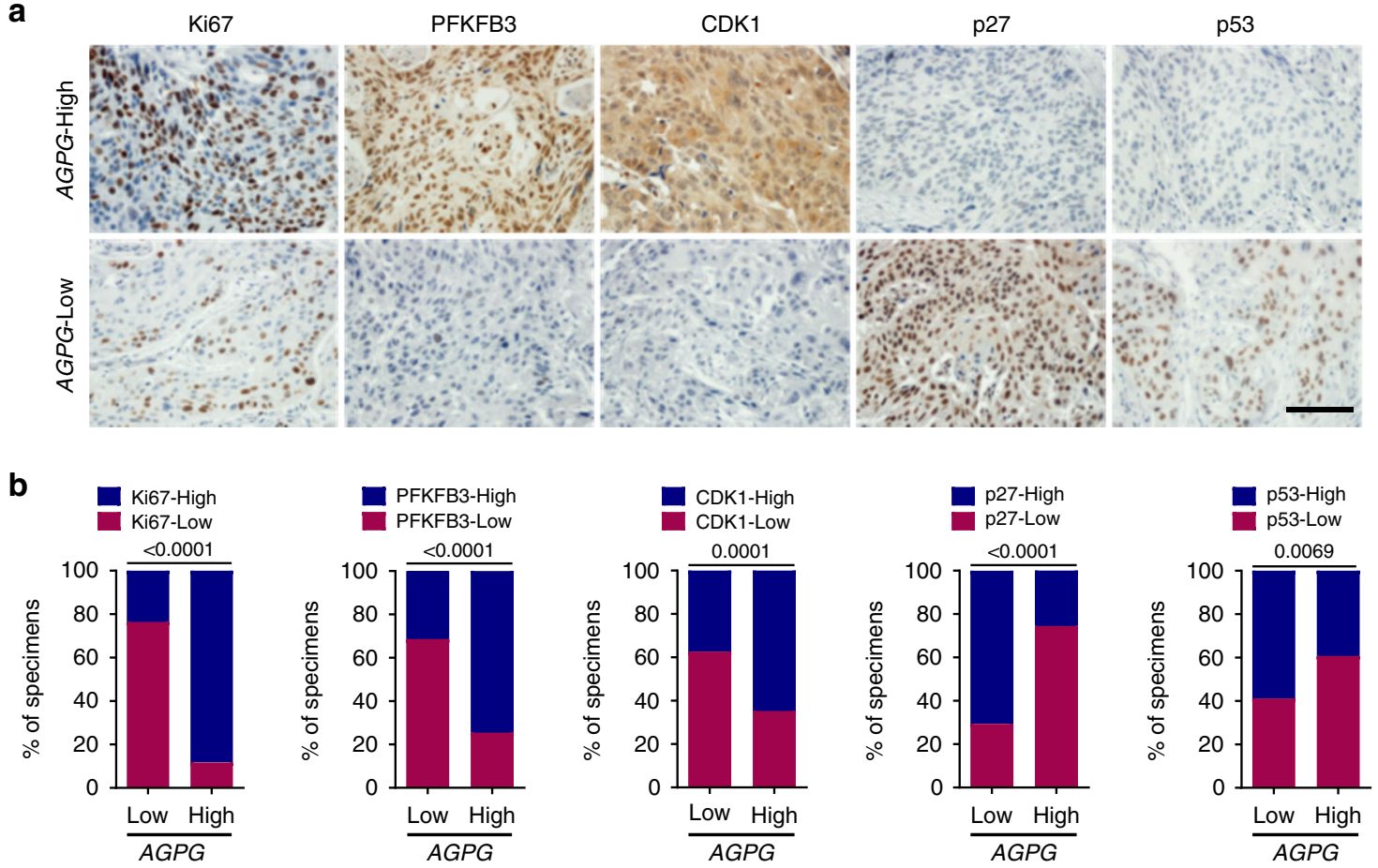

$\mathbf{e}$

- PFKFB3-Low $(n=52)$

g

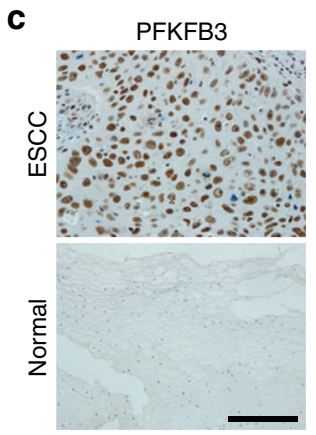

- PFKFB3-High $(n=52)$
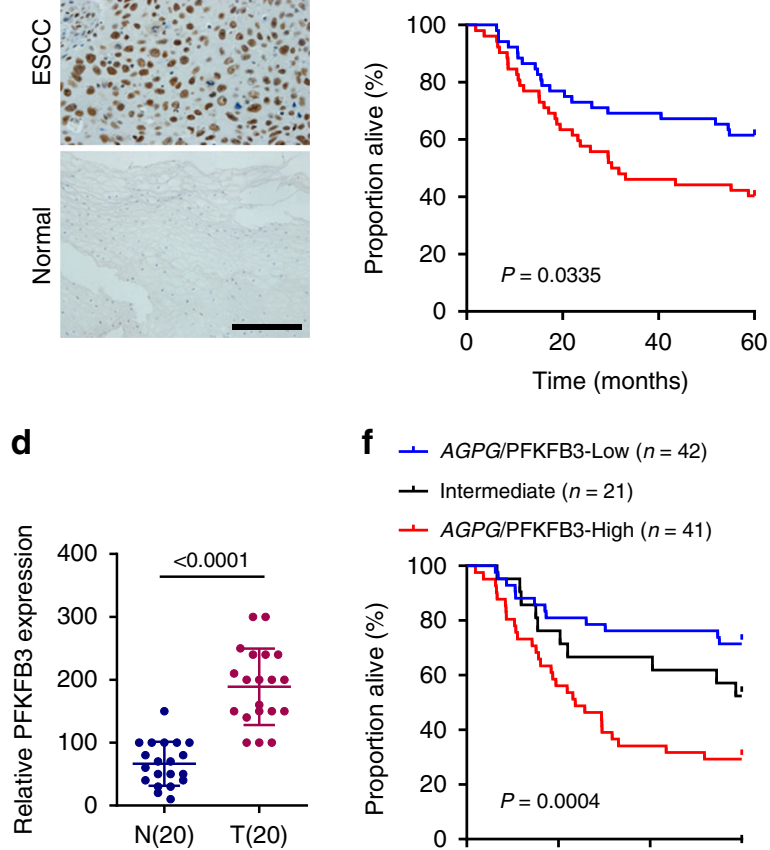

f $\rightarrow$ AGPG/PFKFB3-Low $(n=42)$

$\perp$ Intermediate $(n=21)$

- AGPG/PFKFB3-High $(n=41)$

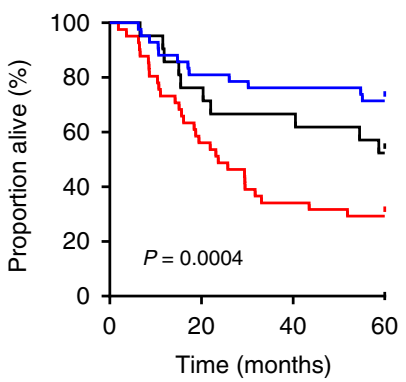

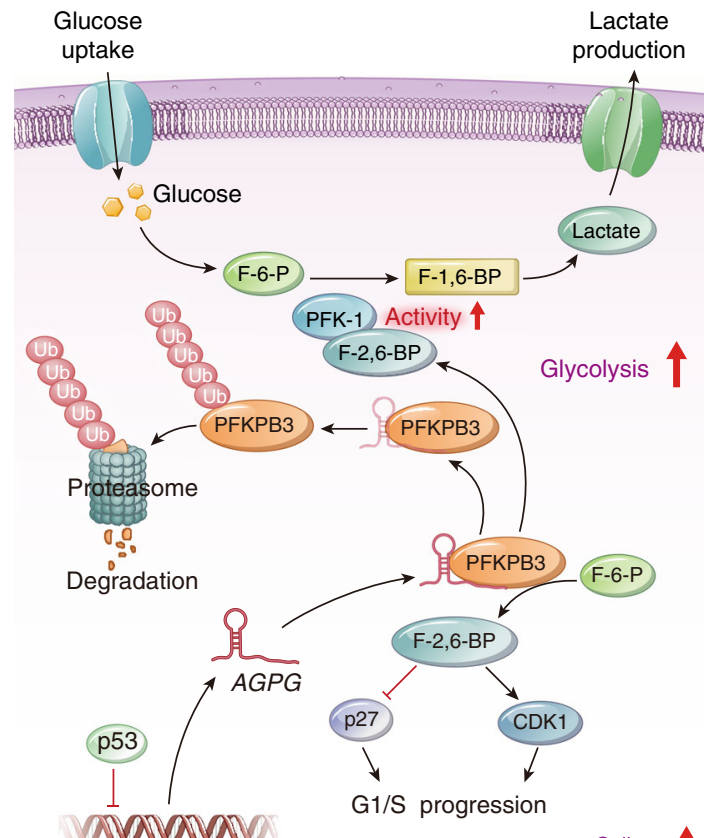

Cell
proliferation

Fig. 7 Clinical relevance of the p53-AGPG-PFKFB3 axis in ESCC. a Representative IHC staining for Ki67, PFKFB3, CDK1, p27, and p53 in ESCC patients (SYSUCC, $n=104$ ) with low or high AGPG expression. Scale bar, $100 \mu \mathrm{m}$. b Percentage of specimens with low or high Ki67, PFKFB3, CDK1, p27, and p53 expression in the low or high AGPG expression groups (SYSUCC, $n=104$, Chi-square test, two-sided). c, d Representative IHC images and statistical analysis of PFKFB3 expression in ESCC and matched normal tissues (SYSUCC). Scale bar, $100 \mu \mathrm{m}$. Data are representative of three independent experiments and presented as mean \pm S.D., $n=20$ cases, the $P$ value was determined by a two-tailed unpaired Student's $t$ test. e Kaplan-Meier analysis of the overall survival of ESCC patients (SYSUCC) with low $(n=52)$ or high $(n=52)$ PFKFB3 expression (log-rank test, two-sided). $\mathbf{f}$ Kaplan-Meier analysis of overall survival of ESCC patients (SYSUCC) with low (low expression of both AGPG and PFKFB3, $n=42$ ), high (high expression of both AGPG and PFKFB3, $n=41)$ or intermediate $(n=21)$ AGPG/PFKFB3 expression (log-rank test, two-sided). $\mathbf{g}$ Graphical abstract showing that the IncRNA AGPG regulates glucose metabolism remodeling by affecting PFKFB3 stability. 
$0.5 \%$ Triton X-100. FISH probes were designed by RiboBio (Guangzhou, China). Hybridization was carried out overnight in a humidified chamber at $37^{\circ} \mathrm{C}$ in the dark. All images were obtained with an Olympus FV1000 confocal microscope (Tokyo, Japan). 4',6-diamidino-2-phenylindole and Cy3 channels were used to detect the signals. $18 \mathrm{~S}$ and U6 were used as the cytoplasmic and nuclear markers, respectively. Immunofluorescence staining was performed according to the manufacturer's instructions, and an anti-PFKFB3 antibody (1:150, Abcam, ab181861) was used in this study.

ChIP, IP, and luciferase reporter assays. ChIP assays were performed with a ChIP kit (Millipore). qPCR analysis was performed to detect the DNA fragments immunoprecipitated with p53. An anti-p53 antibody (1:20, Abcam, ab1101) was used in the ChIP assays. For the IP assays, an anti-PFKFB3 antibody (1:50, Abcam, ab181861) was used, and the immunoprecipitants were detected by western blotting. Luciferase reporter assays were performed according to the manufacturer's instructions (Promega, WI, USA). Firefly and Renilla luciferase activities were examined by the Dual-Luciferase Reporter Assay System, and firefly activity was normalized to Renilla activity.

RNAScope ISH assay and IHC. RNAScope ISH assays were carried out with an RNAScope 2.0 High Definition Assay Kit (Advanced Cell Diagnostics, Newark, CA, USA). A reactive score was obtained according to the percentage of positive cells and the staining intensity. For the IHC assays, staining and analysis were performed according to the manufacturer's instructions ${ }^{53}$. Anti-Ki67 (1:250, Abcam, ab15580), anti-PFKFB3 (1:250, Abcam, ab181861), anti-p27 (1:200, Abcam, ab32034), anti-CDK1 (1:250, Abcam, ab133327), and anti-p53 antibodies (1:200, Abcam, ab1101) were used. For quantification analysis, we evaluated the extent and intensity of all markers.

Cell-based xenograft and PDX models. ESCC cells $\left(2 \times 10^{6}\right)$ expressing shCtrl or sh $A G P G$ were injected subcutaneously into the dorsal flanks of 4 -week-old female $\mathrm{BALB} / \mathrm{c}$ nu/nu mice (five mice per group). Tumor growth was monitored every 3 days after transplantation using calipers. To generate PDX models, fresh ESCC tumor samples from patients were immediately inoculated subcutaneously into both flanks of nude mice. When the successfully established PDXs (P1) reached $\sim 500 \mathrm{~mm}^{3}$, the tumors were transplanted to other mice (P2). Eventually, the mice bearing P3 grafts were used to examine the therapeutic effects of $A G P G$ inhibitor. Twenty-one days after transplantation, we began to perform intratumoral injections of scrambled or in vivo-optimized $A G P G$ inhibitor $(5 \mathrm{nmol}$ per injection, RiboBio, Guangzhou, China) every 3 days. When the study finished, the mice were anesthetized, and the tumor volume and weight were measured. All tissues from the cell-based xenografts or PDXs underwent further pathological analysis.

Statistics and reproducibility. All experiments were carried out at least three times, for RNAScope ISH, FISH, immunofluorescence staining, IHC, and western blot assays, representative images are shown. The results are presented as the mean \pm S.D. of at least three independent experiments after analysis by Student's $t$ test or one-way ANOVA using GraphPad Prism 8.0.1 (GraphPad, La Jolla, CA, USA). Relative gene expression was analyzed using the $2^{-\Delta C t}$ or $2^{-\Delta \Delta C t}$ method. Correlations between AGPG levels and PFKFB3 expression were analyzed with Pearson's correlation analysis. Survival analyses were performed using the Kaplan-Meier method and assessed using the log-rank test. All the statistical tests were two-sided, $P<0.05$ was considered statistically significant.

Study approval. The study was approved by the Medical Ethics Committee of SYSUCC. Written informed consent was obtained from the patients who provided samples. The animal studies were approved by the Institutional Animal Care and Use Committee of Sun Yat-Sen University.

Reporting summary. Further information on research design is available in the Nature Research Reporting Summary linked to this article.

\section{Data availability}

Expression and survival analyses for IncRNAs in ESCC were performed using data obtained from TCGA ${ }^{54}$ (http://www.cbioportal.org/publicportal/). DESeq2 (version 3.10) was employed for differential expression analysis ${ }^{55}$. The pathway analysis was performed with GSEA (version 4.0.3) ${ }^{56}$, whereas JASPAR software (version 2018) predicted the p53binding sequence ${ }^{57}$. The posttranslational modification data of PFKFB3 were obtained from the dbPAF (version 1.0) ) $^{58}$ and CPLM databases (version 1.0) ${ }^{59}$ and visualized by IBS software (version 1.0.3) ${ }^{60}$. The CLIP-Seq data set is available at NCBI Sequence Read Archive (SRA) under BioProject PRJNA591321 (https://www.ncbi.nlm.nih.gov/ bioproject/591321). For CLIP-seq analysis, HomeR (homer2 version) was used to perform the motif analysis on the binding peaks obtained by the Piranha (version 1.2.1) and CIMS (version 1.1.3) analyses. All the other data supporting the findings of this study are available within the article and its Supplementary Information Files or from the corresponding authors upon reasonable request.
Received: 20 December 2018; Accepted: 20 February 2020; Published online: 20 March 2020

\section{References}

1. WEINHOUSE, S. On respiratory impairment in cancer cells. Science 124, 267-269 (1956).

2. Shankaraiah, R. C., Veronese, A., Sabbioni, S. \& Negrini, M. Non-coding RNAs in the reprogramming of glucose metabolism in cancer. Cancer Lett. 419, 167-174 (2018).

3. Chen, F. et al. Extracellular vesicle-packaged HIF-1alpha-stabilizing lncRNA from tumour-associated macrophages regulates aerobic glycolysis of breast cancer cells. Nat. Cell Biol. 21, 498-510 (2019).

4. Ju, H. et al. Modulation of redox homeostasis by inhibition of MTHFD2 in colorectal cancer: mechanisms and therapeutic implications. J. Natl Cancer Inst. 111, 584-596 (2019).

5. Ju, H. et al. ITD mutation in FLT3 tyrosine kinase promotes Warburg effect and renders therapeutic sensitivity to glycolytic inhibition. Leukemia 31, 2143-2150 (2017)

6. Lu, Y. X. et al. ME1 regulates NADPH homeostasis to promote gastric cancer growth and metastasis. Cancer Res. 78, 1972-1985 (2018).

7. Ju, H. Q. et al. Mutant Kras- and p16-regulated NOX4 activation overcomes metabolic checkpoints in development of pancreatic ductal adenocarcinoma. Nat. Commun. 8, 14437 (2017).

8. Branco, C. \& Johnson, R. S. To PFKFB3 or not to PFKFB3, that is the question. Cancer Cell 30, 831 (2016).

9. Yalcin, A. et al. Nuclear targeting of 6-phosphofructo-2-kinase (PFKFB3) increases proliferation via cyclin-dependent kinases. J. Biol. Chem. 284, 24223-24232 (2009).

10. Yalcin, A. et al. 6-Phosphofructo-2-kinase (PFKFB3) promotes cell cycle progression and suppresses apoptosis via Cdk1-mediated phosphorylation of p27. Cell Death Dis. 5, e1337 (2014).

11. Zhu, W. et al. PFK15, a small molecule inhibitor of PFKFB3, induces cell cycle arrest, apoptosis and inhibits invasion in gastric cancer. PloS ONE 11, e163768 (2016).

12. Schoors, S. et al. Partial and transient reduction of glycolysis by PFKFB3 blockade reduces pathological angiogenesis. Cell Metab. 19, 37-48 (2014).

13. Cantelmo, A. R. et al. Inhibition of the glycolytic activator PFKFB3 in endothelium induces tumor vessel normalization, impairs metastasis, and improves chemotherapy. Cancer Cell 30, 968-985 (2016).

14. Mattioli, K. et al. High-throughput functional analysis of lncRNA core promoters elucidates rules governing tissue specificity. Genome Res. 29, 344-355 (2019).

15. Xing, Z. et al. Expression of long noncoding RNA YIYA promotes glycolysis in breast cancer. Cancer Res. 78, 4524-4532 (2018).

16. $\mathrm{Li}, \mathrm{W}$. et al. Increased levels of the long intergenic non-protein coding RNA POU3F3 promote DNA methylation in esophageal squamous cell carcinoma cells. Gastroenterology 146, 1714-1726 (2014).

17. Yamamoto, T. et al. Reduced methylation of PFKFB3 in cancer cells shunts glucose towards the pentose phosphate pathway. Nat. Commun. 5, 3480 (2014).

18. Cabili, M. N. et al. Localization and abundance analysis of human lncRNAs at single-cell and single-molecule resolution. Genome Biol. 16, 20 (2015).

19. Uren, P. J. et al. Site identification in high-throughput RNA-protein interaction data. Bioinformatics 28, 3013-3020 (2012).

20. Zhang, C. \& Darnell, R. B. Mapping in vivo protein-RNA interactions at single-nucleotide resolution from HITS-CLIP data. Nat. Biotechnol. 29, 607-614 (2011).

21. Herrero-Mendez, A. et al. The bioenergetic and antioxidant status of neurons is controlled by continuous degradation of a key glycolytic enzyme by APC/ C-Cdh1. Nat. Cell Biol. 11, 747 (2009).

22. Marsin, A., Bouzin, C., Bertrand, L. \& Hue, L. The stimulation of glycolysis by hypoxia in activated monocytes is mediated by AMP-activated protein kinase and inducible 6-Phosphofructo-2-kinase. J. Biol. Chem. 277, 30778-30783 (2002).

23. Cedeno, C., La Monaca, E., Esposito, M. \& Gutierrez, G. J. Detection and analysis of cell cycle-associated APC/C-mediated cellular ubiquitylation in vitro and in vivo. Methods Mol. Biol. 1449, 251-265 (2016).

24. Ji, J., Wu, K., Wu, M. \& Zhan, Q. P53 functional activation is independent of its genotype in five esophageal squamous cell carcinoma cell lines. Front. Med. China 4, 412-418 (2010).

25. $\mathrm{Hu}, \mathrm{W}$. L. et al. GUARDIN is a p53-responsive long non-coding RNA that is essential for genomic stability. Nat. Cell Biol. 20, 492-502 (2018).

26. Leszczynska, K. B. et al. Hypoxia-induced p53 modulates both apoptosis and radiosensitivity via AKT. J. Clin. Invest. 125, 2385-2398 (2015).

27. $\mathrm{Hu}, \mathrm{Y}$. et al. K-rasG12V transformation leads to mitochondrial dysfunction and a metabolic switch from oxidative phosphorylation to glycolysis. Cell Res. 22, 399-412 (2012). 
28. Yu, X. X. et al. Antisense oligonucleotide reduction of DGAT2 expression improves hepatic steatosis and hyperlipidemia in obese mice. Hepatology 42, 362-371 (2005).

29. Doménech, E. et al. AMPK and PFKFB3 mediate glycolysis and survival in response to mitophagy during mitotic arrest. Nat. Cell Biol. 17, 1304-1316 (2015).

30. Atsumi, T. et al. High expression of inducible 6-phosphofructo-2-kinase/ fructose-2,6-bisphosphatase (iPFK-2; PFKFB3) in human cancers. Cancer Res. 62, 5881-5887 (2002).

31. Jia, W. et al. Non-canonical roles of PFKFB3 in regulation of cell cycle through binding to CDK4. Oncogene 37, 1685-1698 (2018).

32. Yu, L. et al. Modeling the genetic regulation of cancer metabolism: Interplay between glycolysis and oxidative phosphorylation. Cancer Res. 77, 1564-1574 (2017).

33. Li, F. et al. Acetylation accumulates PFKFB3 in cytoplasm to promote glycolysis and protects cells from cisplatin-induced apoptosis. Nat. Commun. 9, 508 (2018).

34. Schmitt, A. M. \& Chang, H. Y. Long noncoding RNAs in cancer pathways. Cancer Cell 29, 452-463 (2016).

35. Ramanathan, M., Porter, D. F. \& Khavari, P. A. Methods to study RNA-protein interactions. Nat. Methods 16, 225-234 (2019).

36. Deben, C. et al. Hypoxia-Induced cisplatin resistance in Non-Small cell lung cancer cells is mediated by HIF- $1 \alpha$ and mutant p53 and can be overcome by induction of oxidative stress. Cancers 10, 126 (2018).

37. Idogawa, M. et al. Identification and analysis of large intergenic non-coding RNAs regulated by p53 family members through a genome-wide analysis of p53-binding sites. Hum. Mol. Genet. 23, 2847-2857 (2014).

38. Huarte, $\mathrm{M}$. et al. A large intergenic noncoding RNA induced by p53 mediates global gene repression in the p53 response. Cell 142, 409-419 (2010).

39. Kawauchi, K., Araki, K., Tobiume, K. \& Tanaka, N. P53 regulates glucose metabolism through an IKK-NF-kappaB pathway and inhibits cell transformation. Nat. Cell Biol. 10, 611-618 (2008).

40. Maddocks, O. D. et al. Serine starvation induces stress and p53-dependent metabolic remodelling in cancer cells. Nature 493, 542-546 (2013).

41. Godar, S. et al. Growth-inhibitory and tumor- suppressive functions of p53 depend on its repression of CD44 expression. Cell 134, 62-73 (2008).

42. Miki, T., Matsumoto, T., Zhao, Z. \& Lee, C. C. P53 regulates Period2 expression and the circadian clock. Nat. Commun. 4, 2444 (2013).

43. Bykov, V. J. N., Eriksson, S. E., Bianchi, J. \& Wiman, K. G. Targeting mutant p53 for efficient cancer therapy. Nat. Rev. Cancer 18, 89 (2017).

44. Brosh, R. \& Rotter, V. When mutants gain new powers: News from the mutant p53 field. Nat. Rev. Cancer 9, 701-713 (2009).

45. Graeber, T. G. et al. Hypoxia-mediated selection of cells with diminished apoptotic potential in solid tumours. Nature 379, 88-91 (1996).

46. Song, Y. et al. Identification of genomic alterations in oesophageal squamous cell cancer. Nature 509, 91 (2014).

47. Kamangar, F., Dores, G. M. \& Anderson, W. F. Patterns of cancer incidence, mortality, and prevalence across five continents: defining priorities to reduce cancer disparities in different geographic regions of the world. J. Clin. Oncol. 24, 2137-2150 (2006).

48. Wang, F. et al. The genomic landscape of small cell carcinoma of the esophagus. Cell Res. 28, 771-774 (2018).

49. van Hagen, P. et al. Preoperative chemoradiotherapy for esophageal or junctional cancer. N. Engl. J. Med. 366, 2074-2084 (2012).

50. Zhuo, W. et al. Long noncoding RNA GMAN, up-regulated in gastric cancer tissues, is associated with metastasis in patients and promotes translation of ephrin a1 by competitively binding GMAN-AS. Gastroenterology 156, 676-691 (2019).

51. Chen, D. L. et al. Long non-coding RNA UICLM promotes colorectal cancer liver metastasis by acting as a ceRNA for microRNA-215 to regulate ZEB2 expression. Theranostics 7, 4836-4849 (2017).

52. Van Nostrand, E. L. et al. Robust transcriptome-wide discovery of RNAbinding protein binding sites with enhanced CLIP (eCLIP). Nat. Methods 13, 508-514 (2016).

53. Wang, C., Wang, J., Chen, Z., Gao, Y. \& He, J. Immunohistochemical prognostic markers of esophageal squamous cell carcinoma: a systematic review. Chin. J. Cancer 36, 65 (2017).

54. Kim, J. et al. Integrated genomic characterization of oesophageal carcinoma. Nature 541, 169-175 (2017).
55. Love, M. I., Huber, W. \& Anders, S. Moderated estimation of fold change and dispersion for RNA-seq data with DESeq2. Genome Biol. 15, 550 (2014)

56. Subramanian, A. et al. Gene set enrichment analysis: a knowledge-based approach for interpreting genome-wide expression profiles. Proc. Natl Acad. Sci. USA 102, 15545-15550 (2005).

57. Khan, A. et al. JASPAR 2018: Update of the open-access database of transcription factor binding profiles and its web framework. Nucleic Acids Res. 46, D260-D266 (2018).

58. Ullah, S. et al. DbPAF: an integrative database of protein phosphorylation in animals and fungi. Sci. Rep. 6, 23534 (2016).

59. Liu, Z. et al. CPLM: a database of protein lysine modifications. Nucleic Acids Res. 42, D531-D536 (2014).

60. Liu, W. et al. IBS: an illustrator for the presentation and visualization of biological sequences. Bioinformatics 31, 3359-3361 (2015).

\section{Acknowledgements}

This research was supported by the Natural Science Foundation of Guangdong Province (2019A1515010233), Fundamental Research Funds for the Central Universities (19ykyjs67), Natural Science Foundation of China (81972239, U1601229), Program for Guangdong Introducing Innovative and Entrepreneurial Teams (2017ZT07S096), Guangdong Esophageal Cancer Institute Science and Technology Program (M201707), Young Teacher Training Program of Sun Yat-Sen University (17ykpy78), and CAMS Innovation Fund for Medical Sciences (2019-I2M-5-036).

\section{Author contributions}

R.-H.X., H.-Q.J., and J.L. designed the study. J.L., Q.-N.W., Y.-X.L., C.-W.W., L.M., Y.W. Z.-X.W., M.-M.H., Y.J., and C.R. collected the data. R.-H.X., H.-Q.J., J.L., Z.-X.L., Q.-N.W., Y.-X.L., C.-W.W., L.M., and Z.-X.W. analyzed and interpreted the data. R.-H.X., H.-Q.J., J.L., Z.-X.L., Q.-N.W., Y.-X.L., and C.-W.W. wrote the manuscript. R.-H.X., J.L., Z.-X.L., Q.-N.W., Y.-X.L., D.-S.W., D.-L.C., H.-Y.P., D.X., M.-S.Z., L.F., B.L., A.L., P.H., D.-X.L., and H.-Q.J. revised the manuscript. R.-H.X., H.-Q.J., J.L., Z.-X.L. Q.-N.W., Y.-X.L., C.-W.W., and L.M. performed the statistical analysis. All authors reviewed the manuscript and approved the final version.

\section{Competing interests}

The authors declare no competing interests.

\section{Additional information}

Supplementary information is available for this paper at https://doi.org/10.1038/s41467020-15112-3.

Correspondence and requests for materials should be addressed to R.-H.X. or H.-Q.J.

Peer review information Nature Communications thanks the anonymous reviewer(s) for their contribution to the peer review of this work. Peer reviewer reports are available.

Reprints and permission information is available at http://www.nature.com/reprints

Publisher's note Springer Nature remains neutral with regard to jurisdictional claims in published maps and institutional affiliations.

Open Access This article is licensed under a Creative Commons Attribution 4.0 International License, which permits use, sharing, adaptation, distribution and reproduction in any medium or format, as long as you give appropriate credit to the original author(s) and the source, provide a link to the Creative Commons license, and indicate if changes were made. The images or other third party material in this article are included in the article's Creative Commons license, unless indicated otherwise in a credit line to the material. If material is not included in the article's Creative Commons license and your intended use is not permitted by statutory regulation or exceeds the permitted use, you will need to obtain permission directly from the copyright holder. To view a copy of this license, visit http://creativecommons.org/ licenses/by/4.0/

(C) The Author(s) 2020 